\title{
What is an archaeon and are the archaea really unique?
}

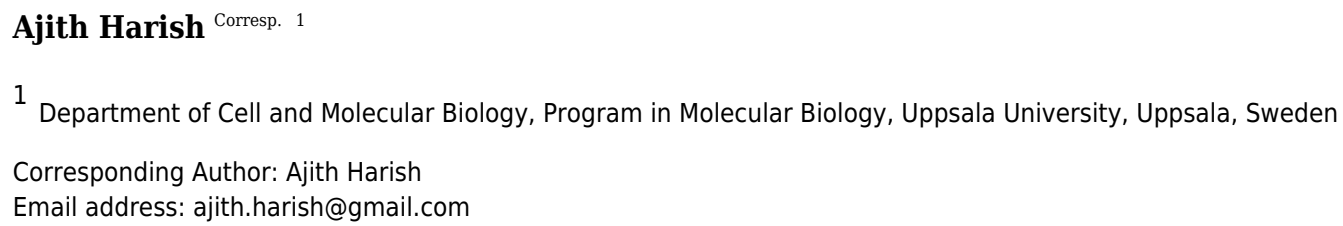

The recognition of the group Archaea as a major branch of the Tree of Life (ToL) prompted a new view of the evolution of biodiversity. The genomic representation of archaeal biodiversity has since significantly increased. In addition, advances in phylogenetic modeling of multi-locus datasets have resolved many recalcitrant branches of the ToL. Despite the technical advances and an expanded taxonomic representation, two important aspects of the origins and evolution of the Archaea remain controversial, even as we celebrate the 40th anniversary of the monumental discovery. These issues concern (i) the uniqueness (monophyly) of the Archaea, and (ii) the evolutionary relationships of the Archaea to the Bacteria and the Eukarya; both of these are relevant to the deep structure of the ToL. To explore the causes for this persistent ambiguity, I examine multiple datasets and different phylogenetic approaches that support contradicting conclusions. I find that the uncertainty is primarily due to a scarcity of information in standard datasets-universal core genes datasets-to reliably resolve the conflicts. These conflicts can be resolved efficiently by comparing patterns of variation in the distribution of functional genomic signatures, which are less diffused unlike patterns of primary sequence variation. Relatively lower heterogeneity in distribution patterns minimizes uncertainties and supports statistically robust phylogenetic inferences, especially of the earliest divergences of life. This case study further highlights the limitations of primary sequence data in resolving difficult phylogenetic problems, and raises questions about evolutionary inferences drawn from the analyses of sequence alignments of a small set of core genes. In particular, the findings of this study corroborate the growing consensus that reversible substitution mutations may not be optimal phylogenetic markers for resolving early divergences in the ToL, nor for determining the polarity of evolutionary transitions across the ToL. 


\section{What is an Archaeon and are the Archaea Really Unique?}

2 Ajith Harish ${ }^{*}$

$3{ }^{1}$ Department of Cell and Molecular Biology, Program in Molecular Biology, Uppsala

4 University, Uppsala, Sweden.

5 Corresponding Author: Ajith Harish

6 E-mail address: ajith.harish@gmail.com

\section{ABSTRACT}

9 The recognition of the group Archaea as a major branch of the Tree of Life (ToL) prompted a new view of the evolution of biodiversity. The genomic representation of archaeal biodiversity has since significantly increased. In addition, advances in phylogenetic modeling of multi-locus datasets have resolved many recalcitrant branches of the ToL. Despite the technical advances and an expanded taxonomic representation, two important aspects of the origins and evolution of the Archaea remain controversial, even as we celebrate the 40th anniversary of the monumental discovery. These issues concern (i) the uniqueness (monophyly) of the Archaea, and (ii) the evolutionary relationships of the Archaea to the Bacteria and the Eukarya; both of these are relevant to the deep structure of the ToL. To explore the causes for this persistent ambiguity, I examine multiple datasets and different phylogenetic approaches that support contradicting conclusions. I find that the uncertainty is primarily due to a scarcity of information in standard datasets - universal core genes datasets - to reliably resolve the conflicts. These conflicts can be resolved efficiently by comparing patterns of variation in the distribution of functional genomic signatures, which are less diffused unlike patterns of primary sequence variation. Relatively lower heterogeneity in distribution patterns minimizes uncertainties and supports statistically robust phylogenetic inferences, especially of the earliest divergences of life. This case study further highlights the limitations of primary sequence data in resolving difficult phylogenetic problems, and raises questions about evolutionary inferences drawn from the analyses of sequence alignments of a small set of core genes. In particular, the findings of this study corroborate the growing consensus that reversible substitution mutations may not be optimal phylogenetic markers for resolving early divergences in the ToL, nor for determining the polarity of evolutionary transitions across the ToL. 
32

33

34

35

36

37

38

39

40

41

42

43

44

45

46

47

48

49

\section{INTRODUCTION}

The recognition of the Archaea as the so-called "third form of life" was made possible in part by a new technology for sequence analysis, oligonucleotide cataloging, developed by Fredrik Sanger and colleagues in the 1960s (Woese 2004; Woese \& Fox 1977). Carl Woese's insight of using this method, and the choice of the small subunit ribosomal RNA (16S/SSU rRNA) as a phylogenetic marker, not only put microorganisms on a phylogenetic map (or tree), but also revolutionized the field of molecular systematics that Zukerkandl and Pauling had previously alluded to (Zuckerkandl \& Pauling 1965). Comparative analysis of organism-specific oligonucleotide signatures in SSU rRNA led to the recognition of a distinct group of microorganisms (Woese 2004). Initially referred to as Archaeabacteria, SSU rRNAs in these unusual organisms had 'sequence signatures' distinct from other bacteria (Eubacteria), and they were later found to be different from those of eukaryotes (Eukarya) as well. Many other signatures, including molecular, biochemical as well as ecological features, corroborated the uniqueness of the Archaea. Thus the archaeal concept was established (Woese 2004). Accordingly, the Five-Kingdoms of life classification scheme (Whittaker 1969) was replaced by the Three-Domains of life classification (Woese et al. 1990).

The genomic representation of microbial biodiversity, particularly of the Archaea, has since expanded significantly. This is largely due to advances in environmental genome sequencing: the sampling of microbial DNA directly from the environment without the need for culturing (Rinke et al. 2013; Sunagawa et al. 2015). Since large-scale exploration by the means of environmental genome sequencing became possible almost a decade ago, there has also been a palpable excitement and anticipation of the discovery of a fourth form of life or a "fourth domain" of life (Wu et al. 2011). The reference here is to a fourth form of cellular life, but not to viruses, which some have already proposed to be the fourth domain of the Tree of Life (ToL) (Boyer et al. 2010). If a fourth form of life were to be found, what would the distinguishing features be, and how could it be measured, defined and classified?

Rather than the discovery of a fourth domain, and contrary to the expectations, however, current discussion is centered around the return to a dichotomous classification of life (Harish \& Kurland 2017a; Harish et al. 2013; Williams et al. 2013); despite hundreds of novel phyla descriptions (Hug et al. 2016; Parks et al. 2017). The proposed dichotomous classification schemes, however, are in sharp contrast to each other, depending on: (i) whether the Archaea 
63 constitute a monophyletic group - a unique line of descent that is distinct from those of the 64 Bacteria as well as the Eukarya; and (ii) whether the Archaea form a sister clade to the Eukarya or to the Bacteria. Both the issues stem from difficulties involved in resolving the deep branches of the ToL (Gouy et al. 2015; Harish et al. 2013; Williams et al. 2013).

The twin issues, first recognized based on discordant tree topologies derived from singlegene analyses (Lake 1986; Tourasse \& Gouy 1999), continue to be the subjects of a longstanding debate, which remains unresolved despite large-scale analyses of multi-gene datasets (Da Cunha et al. 2017; Rinke et al. 2013; Spang et al. 2015; Williams \& Embley 2014; ZarembaNiedzwiedzka et al. 2017). In addition to the choice of genes to be analyzed, the choice of the underlying character evolution-model is at the core of contradictory results that either support the Three-Domains tree in which the Archaea are monophyletic and sister to Eukarya (Da Cunha et al. 2017; Rinke et al. 2013; Woese et al. 1990); or the Eocyte tree, in which the Archaea are paraphyletic, and some archaea (Crenarchaea/eocytes) are sister to Eukarya (Lake et al. 1984; Williams \& Embley 2014; Zaremba-Niedzwiedzka et al. 2017). A third competing hypotheses is the Two-Empires tree, which places Archaea sister to Bacteria (Brinkmann \& Philippe 1999; Mayr 1998), but is rarely considered. In many cases of such systematic discordances, adding more data, either as enhanced taxon sampling or enhanced character sampling, or both, can resolve ambiguities (Salichos \& Rokas 2013; Zwickl \& Hillis 2002). However, as the taxonomic diversity and evolutionary distance increases among the taxa studied, the number of conserved marker-genes that can be aligned for phylogenetic analyses decreases. Accordingly, recovery of the historical signal in multiple sequence alignments (MSAs) by phylogenetic analyses is restricted to a small set of conserved loci or genes - 50 at most (Zaremba-Niedzwiedzka et al. 2017) — usually referred to as 'universal core genes' (Williams et al. 2013; Woese 2002). is the standard molecular phylogenetic approach. However, several conserved genomic loci, i.e. the loci per se, are a distinct class of phylogenetic markers (Hillis 1999; Rokas \& Holland 2000). Phylogenetic signal can be recovered from covariation patterns among genomes of highly conserved loci such as introns, mobile elements, protein-coding and non-coding genes, proteindomains and other genomic features (Harish et al. 2013; Hillis 1999; Snel et al. 1999; Tarver et al. 2013; Wang \& Caetano-Anolles 2006; Yang et al. 2005). Genomic features are underutilized in phylogenomic studies, even though their advantages over single-residue substitutions, for 
94 instance, low observed levels of homoplasy, has been known for long. This was, initially, due to

95 the practical difficulties in collecting multiple characters per clade, and over a broad range of

96 taxonomic groups, to assemble large-scale datasets that is necessary for statistically robust

97 inferences (Hillis 1999). Although assembling large datasets is no longer a barrier for the

98 estimation of phylogeny using genomic features, it was, until recently, limited to parsimony

99 methods (Harish et al. 2013; Kim \& Caetano-Anollés 2011). Analysis of datasets with hundreds

100 of taxa is now feasible using both maximum likelihood (Fang et al. 2013) as well as Bayesian

101 (Harish \& Kurland 2017a) methods, but the statistical behavior and robustness to rate

102 heterogeneities have not yet been carefully characterized.

103 Altogether, independent phylogenomic analyses that employ different, but overlapping

104 datasets yield alternative tree topologies with incompatible branching patterns (Da Cunha et al.

105 2017; Harish \& Kurland 2017a; Kim \& Caetano-Anollés 2011; Rinke et al. 2013; Spang et al.

106 2015; Williams \& Embley 2014). Contradicting conclusions are also supported when different

107 analytical approaches are applied to the same datasets (Da Cunha et al. 2017; Harish \& Kurland

108 2017a; Rinke et al. 2013; Spang et al. 2015; Williams \& Embley 2014). Despite the

109 contradictions, the branches typically receive high branch support values - statistical measures of

110 confidence in a given branch - and thus provide equivocal support for contradicting scenarios for

111 the early diversification of Archaea.

112 Here, to understand the source of such conflicting results, I examine different

113 phylogenomic datasets and alternative approaches used to resolve such conflicts. Specifically,

114 the quality of different types of molecular features, and the utility or a lack there of, of such data

115 for resolving complex phylogenetic problems is assessed. I find that a primary cause for this

116 persistent ambiguity is that the 'information' necessary to resolve these conflicts is inadequate in

117 the standard 'universal core genes' datasets employed routinely to reconstruct the global ToL. In

118 contrast, covariation patterns of unique genomic loci provide for sufficient information for a

119 reliable resolution of the conflicts. Resolving the evolutionary relationships of archaea to other

120 taxa, however, depends on the placement of the root of the ToL (Brinkmann \& Philippe 1999;

121 Harish et al. 2013). Using an expanded taxonomic sampling of recently described groups of

122 archaea, including the TACK, DPANN and Asgard archaea (Zaremba-Niedzwiedzka et al.

123 2017), I re-evaluate the utility of directional evolution-models (Harish \& Kurland 2017a;

124 Klopfstein et al. 2015) to identify the root of the ToL. I find that the resolution of the 
125 phylogenetic radiations, in deep time, based on genomic features is robust against potential

126 artifacts due to biases in character specific and lineage specific rate heterogeneity (heterotachy)

127 as well as composition bias.

128 Accordingly, phylogenetic modeling of the evolution of genomic features validates the

129 uniqueness (monophyly) of the Archaea, and the placement of Archaea sister to Bacteria

130 (Brinkmann \& Philippe 1999; Harish \& Kurland 2017b; Harish et al. 2013; Mayr 1998). Further,

131 the independent and parallel diversification of eukaryote and akaryote species is corroborated

132 (Forterre \& Philippe 1999; Harish \& Kurland 2017a; Harish \& Kurland 2017c). Findings from

133 this case study on Archaea are broadly applicable to the problem of incongruence that is often

134 encountered in efforts to resolve certain other early divergences in the ToL, for example, at the

135 root of the eukaryote-ToL (Derelle et al. 2015; He et al. 2014) and the metazoan-ToL (Philippe

136 et al. 2011; Shen et al. 2017; Whelan et al. 2015).

137 Importantly this study shows that, despite the presence of conflicting signals that arise

138 from disparate processes of reticulate evolution, the earliest divergences of life can be

139 reconstructed reliably using genomic signatures of evolutionary transitions. I discuss

140 underutilized approaches to recover phylogenetic signal in genome sequence data that are

141 valuable to minimize phylogenetic uncertainties. Finally, I discuss simple but important, yet

142 undervalued, aspects of phylogenetic hypothesis testing, which together with the new approaches

143 hold promise to resolve these long-standing issues effectively.

\section{DATA AND METHODS}

\section{Data Sources and Data (Character) Types Analyzed}

147 Five datasets, one single-locus dataset and four multi-locus phylogenomic datasets were 148 analyzed in this study (Table 1). All datasets, except one, were obtained from previous studies 149 that focused on resolving the phylogenetic relatedness of Archaea to Eukarya and Bacteria. A 150 new dataset was assembled for this study, to include recently discovered taxa (see details below).

151 To distinguish the different character codings used to represent genomic loci in the data matrices, 152 characters are classified as either (i) Elementary molecular characters: Single-residue (nucleotide 153 and amino acid) characters in MSA; or, (ii) Complex molecular characters: Genomic features

154 that are distinct permutations of elementary characters. In this study, complex characters are 155 genomic loci that correspond to protein-domains; specifically, domains that are identified from 
156 experimentally determined three-dimensional (3D) structures according to the SCOP (Structural

157 Classification of Proteins) scheme for identifying homologous domains (Gough et al. 2001;

158 Murzin et al. 1995). A detailed description of the different datasets is as follows,

159 (i) Elementary character datasets: MSA datasets were obtained as-is from previous

160 studies (Table 1); a single-gene nucleotide MSA of the SSU rRNA and two amino acid MSAs of

161 concatenated universal core genes. The universal core genes (henceforth simply core-genes) are

162 conserved genes that are found in all organisms, which function in the transcription and

163 translation processes of gene expression. Genes that are included in phylogenomic data matrices

164 mainly encode components of the translation apparatus, ribosomal proteins and translation

165 factors as well as a few components of RNA polymerases. Different MSAs with overlapping sets

166 of core-genes were obtained (Table 1): (a) Core-genes-I dataset is a MSA of 29 genes (Williams

167 \& Embley 2014); (b) Core-genes-II dataset is a MSA of 48 genes (Zaremba-Niedzwiedzka et al.

168 2017). The number of core-genes sampled or the extent of overlap between different datasets

169 depends on taxon sampling and the criteria applied for filtering data to be analyzed (Williams \&

170 Embley 2014). For instance, different sequence similarity thresholds used to identify orthologs,

171 or the level of stringency applied to the definition of universal markers: either to be present in

172 every taxon sampled (universal) or to allow for gene absences to be coded as missing data

173 (nearly universal). Together, these criteria determine the size of the data matrix in terms of the

174 number of characters considered to be informative to test phylogenetic hypotheses (Table 1).

175 (ii) Complex character datasets: Homologous protein-domains were coded with

176 nonarbitrary presence - absence state labels (Lewis 2001). Data matrices of SCOP-domains were

177 assembled from genome annotations available through the SUPERFAMILY HMM library and

178 genome assignments server, v. 1.75 (http://supfam.org/SUPERFAMILY/) (Gough et al. 2001;

179 Oates et al. 2015). When genome annotations were unavailable from the SUPERFAMILY

180 database, curated reference proteomes were obtained from the universal protein resource

181 (http://www.uniprot.org/proteomes/). SCOP-domains were annotated using the HMM library and

182 genome annotation tools as recommended by the SUPERFAMILY resource. A more detailed

183 description of the protocol can be found in (Harish et al. 2013). Two datasets (Table 1) with

184 overlapping taxon samples were assembled as follows,

185 (a) SCOP-I dataset: A 141-species dataset was obtained from a previous study (Harish et al.

186 2013). The broadest possible taxonomic diversity of sequenced genomes available at the time 
187 was sampled. An equal number of species, 47 each, were sampled from Archaea, Bacteria and

188 Eukarya. The number of genomes was limited by the number of unique genera of Archaea for

189 which genome sequences were available at the time of the study. 1,732 of the 2,000 distinct

190 SCOP-domains are represented in this sampling.

191 (b) SCOP-II dataset: The 141-species dataset was updated with representatives of novel species

192 described recently, largely with archaeal species from the TACK group (Guy \& Ettema 2011),

193 DPANN group (Rinke et al. 2013) and Asgard group including the Lokiarchaeota (Zaremba-

194 Niedzwiedzka et al. 2017). In addition, species sampling was enhanced with representatives from

195 the (unclassified) candidate phyla described for bacterial species (Anantharaman et al. 2016) and

196 with unicellular species of eukaryotes, to a total of 222 species. 1,738 SCOP-domains are

197 represented in this sampling. The complete list of the species with their respective Taxonomy

198 IDs is available in Supplementary Table S1. Unlike with curating MSA datasets, data (character)

199 filtering is not required to assemble protein-domain datasets.

200

201

\section{Exploratory Data Analysis}

202

Data Display Networks (DDNs) were constructed with SplitsTree 4.1 (Huson \& Bryant

203

2006). Split networks were computed using the Neighbor-Net method from the observed genetic distances (p-distances) of the taxa for both nucleotide- and amino acid characters in the core-

205 genes datasets. Split networks of the protein-domain characters were computed from Hamming

206 distance. The network diagrams were drawn with the equal angle algorithm.

207

208

\section{Phylogenetic Analyses}

209

Core-genes datasets. - The best-fitting amino acid substitution model was chosen using

210 Smart Model Selection (SMS) (Lefort et al. 2017) and ModelFinder (Kalyaanamoorthy et al.

211 2017). Both model selection tests chose the LG model of amino acid substitution (Le \& Gascuel

212 2008) to be the best fitting model, for both core-genes datasets (Supplementary Table S2 and

213 S3). However, analysis here was restricted to the core-genes-I dataset due to a relatively smaller

214 taxon sampling (44 species) compared to the core-genes-II dataset (96 species), since the

215 computational time required for estimating trees is significantly lesser. Moreover, the general

216 conclusions, including paraphyly of archaea, based on these datasets are consistent (Williams \& 
217 Embley 2014; Zaremba-Niedzwiedzka et al. 2017). Extensive analyses of these two concatenated 218 core-genes datasets are reported in the original studies.

219 Unrooted (undirected) trees were estimated with both the rate-homogeneous as well as 220 rate-heterogeneous versions of the LG model implemented in PhyML 3.0 (Guindon et al. 2010).

221 Character-specific rate heterogeneity (CSRH) was approximated using the discrete gamma 222 distribution (Yang \& Roberts 1995) with 4, 8 and 12 rate categories, LG+G4, LG+G8 and 223 LG+G12, respectively. More complex models (Supplementary Table S2) that account for 224 invariable characters $(\mathrm{LG}+\mathrm{GX}+\mathrm{I})$ and/or models that compute alignment-specific character-state 225 frequencies $(\mathrm{LG}+\mathrm{GX}+\mathrm{F})$ were also used, but the trees inferred were identical to trees estimated 226 from LG+GX models, and therefore not reported here. Log likelihood ratio (LLR) was calculated 227 as the difference in the raw log likelihood scores for each model.

228 SCOP-domain datasets. -Both unrooted (undirected) trees and intrinsically rooted 229 (directed) trees were estimated. The Mk model (Lewis 2001) applicable to complex features 230 coded as binary-state characters is the most widely implemented model for phylogenetic 231 inference in both maximum likelihood (ML) and Bayesian phylogenetic methods. However, only 232 reversible models are implemented in ML software at present. Both reversible and directional 233 evolution-models as well as model selection routines are implemented in MrBayes 3.2

234 (Klopfstein et al. 2015; Ronquist et al. 2012). Directional evolution refers to either

235 nonreversibility of character transitions or nonstationarity of state frequencies, or both, along the 236 tree. Since standard reversible models assume stationarity of character frequencies and 237 reversibility of character transitions, the likelihood scores are independent of the placement of 238 the root. Directional evolution-models, however, relax the standard assumptions to allow 239 nonstationarity and nonreversibility of character transitions such that frequency of characters at 240 the root of the tree is allowed to be different from the rest of tree (Klopfstein et al. 2015).

241 Therefore, likelihood scores depend on the placement of the root.

242

\section{Root Inference}

244 The placement of the root is crucial to determine the monophyly (or non-monophyly) of a 245 taxonomic group as well as sister-group relationships. Several methods can be used to identify 246 the root of phylogenetic trees: Paleontological (temporal) data, outgroup rooting, the molecular 247 clock and directional evolution-models (Huelsenbeck et al. 2002). The former two are not 
248 applicable to the global $\mathrm{ToL}$ as there are no known fossils or outgroups that can be employed.

249 Directional models are able to identify the correct rooting of trees without the use of an outgroup

250 or other prior knowledge (Huelsenbeck et al. 2002; Klopfstein et al. 2015). The utility and

251 efficacy of the directional evolution-model, to detect nonstationarity and nonreversibility is

252 rigorously characterized with simulations and empirical datasets in previous studies (Harish \&

253 Kurland 2017a; Klopfstein et al. 2015). The utility of directional evolution-models to root the

254 global ToL, and the suitability of models that are both nonstationary and nonreversible has been

255 tested and discussed extensively in previous studies using the SCOP-I dataset (Harish \& Kurland

256 2017a; Harish \& Kurland 2017b). In this study, the placement of the root is analyzed further

257 using both non-clock and relaxed-clock models using the SCOP-II dataset (i.e. with an expanded

258 taxonomic diversity)

259

260 Robustness of Root Placement

261 Robustness of root placement against potential systematic biases with focus on errors due

262 to CSRH as well as LSRH (lineage-specific rate heterogeneity or heterotachy) was analyzed in

263 this study. Robustness of root placement against other potential errors was assessed extensively,

264 and reported in four earlier studies (see Supplementary Methods). Briefly, these include impact

265 of: (1) species (taxon) sampling, (2) inclusion and exclusion of lineage-specific domains

266 (characters), (3) small (or large) genome-size bias, (4) uncertainty in domain assignments with

267 HMM models (ascertainment bias) and (5) quality of genome sequence data/annotations.

268 In the present study, sensitivity of the directional model to CSRH was analyzed by

269 varying the number of rate categories under the Gamma rate variation model. In addition, to test

270 if the placement of the root is biased due to LSRH, relaxed-clock models implemented in

271 MrBayes were used under nonstationarity. Relaxed-clock models allow rates to vary across

272 lineages, in addition to rate variation across characters. Three different relaxed clock-models

273 where the rate variation across lineages is modeled according to Compound Poisson Process

274 (CPP) model, Brownian motion model (TK02) and Independent Gamma Rate (IGR) model, with

275 default priors for branch lengths were used (for details see Supplementary Methods).

$276 \quad$ Altogether, 15 different models of increasing complexity that assume (1) rate

277 homogeneity or different extents of CSRH, LSRH; (2) reversibility or nonreversibility, and (3)

278 stationarity or nonstationarity of the evolutionary process were characterized, using the SCOP-II 
279 dataset. The model complexity is proportional to the number of assumptions incorporated in the 280 model. In each case, two independent runs of Metropolis-Coupled MCMC samplings were used

281 with four chains each, sampling every 500th generation. MCMC sampling was run until

282 convergence, unless mentioned otherwise. Convergence was assessed through the average

283 standard deviation of split frequencies (ASDSF, less than 0.01) for tree topology and the

284 potential scale reduction factor (PSRF, equal to 1.00) for scalar parameters, unless mentioned

285 otherwise. The first half of the generations was discarded as burn-in. Bayes factors for model

286 comparison were calculated using the harmonic mean estimator in MrBayes. The log Bayes

287 factor (LBF) was calculated as the difference in the marginal log likelihoods for each model.

288 Convergence between independent runs was generally slower for directional models

289 compared to the reversible models. When convergence was extremely slow (requiring more than

290100 million generations and/or more than 21 days run-time) topology constraints based on the

291 clusters derived from the unrooted trees were applied to improve convergence rates. As such

292 these clusters/constraints corresponded to named taxonomic groups e.g. Fungi, Metazoa,

293 Crenarchaeota, etc. Convergence assessment between independent runs was relaxed for 3 (out of

294 15) models that did not converge sufficiently at the time of submission: non-clock rooted trees

295 corresponding to root-R2 (ASDSF 0.05; PSRF 1.04), root-R3 (ASDSF 0.02; PSRF 1.01) and

296 relaxed-clock rooted tree using the CPP model (ASDSF 0.03; PSRF 1.05). In these three cases

297 specified, the difference in bipartitions is in the shallow parts (minor branches) of the tree, but

298 not within the deeper nodes (major branches). For assessing well-supported major branches of

299 the tree, ASDSF values between 0.01 and 0.05 may be adequate, as recommended by the authors

300 (Ronquist et al. 2011).

301

302 RESULTS

303 Information in Core-genes Datasets is Inadequate to Resolve the Archaeal Radiation

304 Data-display networks (DDNs) are useful to examine and visualize character conflicts in

305 phylogenetic datasets, especially in the absence of prior knowledge about the source of such

306 conflicts (Huson \& Bryant 2006; Morrison 2009). While congruent data will be displayed as a

307 tree in a DDN, incongruences are displayed as reticulations in the tree. Figure 1A shows a

308 neighbor-net analysis of the SSU rRNA alignment used to resolve the phylogenetic position of

309 the recently discovered Asgard archaea (Zaremba-Niedzwiedzka et al. 2017). The DDN is based 
310 on character distances calculated as the observed genetic distance (p-distance) of 1,462

311 characters, and shows the total amount of conflict in the dataset that is incongruent with

312 character bipartitions (splits). The edge (branch) lengths in the DDN correspond to the support

313 for the respective splits. Accordingly, two well-supported sets of splits for the Bacteria and the

314 Eukarya are observed. The Archaea, however, does not form a distinct, well-resolved/well-

315 supported group, and is unlikely to correspond to a monophyletic group in a phylogenetic tree.

316 Likewise, the concatenated protein sequence alignment of the so-called 'genealogy

317 defining core of genes' (Woese 2002) - a set of conserved single-copy genes - also does not

318 support a unique archael lineage. Figure 1B is a DDN derived from a neighbor-net analysis of

319 8,563 characters in 29 concatenated core-genes (Williams \& Embley 2014), while those in

320 Figure $1 \mathrm{C}, \mathrm{D}$ are based on 9,868 characters in 48 concatenated core-genes (also from (Zaremba-

321 Niedzwiedzka et al. 2017)). However, in Figure 1D, amino acids in the MSA are recoded as a

322 reduced set of alphabets using the SR-4 (from 20 to 4) recoding scheme (Susko \& Roger 2007).

323 Even taken together, none of the standard marker gene datasets are likely to support the

324 monophyly of the Archaea - a key assertion of the Three-Domains hypothesis (Woese et al.

325 1990). Simply put, there is not enough information in the core-genes datasets to resolve the

326 archaeal radiation, or to determine whether the Archaea are really unique compared to the

327 Bacteria and Eukarya. However, other complex features — including molecular, biochemical

328 and phenotypic characters, as well as ecological adaptations - support the uniqueness of the

329 Archaea (Garrett 1985; Valentine 2007; Woese 2004).

330

331 Complex Molecular Characters Minimize Uncertainties Regarding the Uniqueness of the

332 Archaea

333 A nucleotide is the smallest possible locus, and an amino acid is a proxy for a locus of a

334 nucleotide triplet. Unlike the elementary amino acid- or nucleotide-characters in the core-genes

335 dataset (Fig.1), the DDN in Figure 2 is based on complex molecular characters: relatively larger

336 genomic loci that are formed by distinct permutations of elementary characters. In this case the

337 loci correspond to protein-domains, typically 200 amino acids (600 nucleotides) long. Each

338 protein-domain is unique: with a distinct sequence profile, 3D structure and function (Fig. 3).

339 Neighbor-net analysis of protein-domain data coded as binary characters (presence/absence) is 
340 based on the Hamming distance (identical to the p-distance used in Fig. 1). Here the Archaea

341 also form a distinct well-supported cluster, as do the Bacteria and the Eukarya.

342 Figure 2A is a DDN based on the dataset that includes protein-domain cohorts of 141

343 species, used in a phylogenomic analysis to resolve the uncertainties at the root of the ToL

344 (Harish \& Kurland 2017a). Compared to the data in Figure 1, the taxonomic diversity sampled

345 for the Bacteria and Eukarya is more extensive, but less extensive for the Archaea; it is

346 composed of the traditional groups Euryarchaeota and Crenarchaeota. Figure 2B is a DDN of an

347 enriched sampling of 81 additional species, which includes representatives of the newly

348 described archaeal groups: TACK (Thaumarchaeota, Aigarchaeota, Crenarchaeota and

349 Korarchaeota), DPANN (Diapherotrites, Parvarchaeota, Aenigmarchaeota, Nanoarchaeota,

350 Nanohaloarchaea), and Asgard (Lokiarchaeota, Thorarchaeota, Odinarchaeota,

351 Heimdallarchaeota). In addition, species sampling was enhanced with representatives from the

352 candidate phyla described for Bacteria, and with unicellular species of Eukarya. The complete

353 list of species analyzed is in Supplementary Table S1.

354 Notably, the extension of the protein-domain cohort is insignificant, from 1,732 to 1,738

355 distinct domains (characters). Based on the well-supported splits in the DDN that form a distinct

356 archaeal cluster, the Archaea are likely to be a monophyletic group (or a clade) in phylogenies

357 inferred from these datasets.

358

359

360

Employing Complex Molecular Characters Maximizes the Representation of Orthologous Non-recombining Genomic Loci, and thus Phylogenetic Signal

361

Despite the superficial similarity of the DDNs in Figures 1 and 2, they are both

362 qualitatively and quantitatively different codings of genome sequences. As opposed to tracing

363 the history of, at most 50 loci, in the standard core-genes datasets (Fig. 1), up to 30 fold more

364 information (1,738 loci) is represented when genome sequences are coded as protein-domain

365 characters (Fig. 2). Currently $\sim 2,000$ unique domains are described by SCOP (Andreeva et al.

366 2014). The phyletic distribution of 1,738 distinct domains identified in the 222 representative

367 species sampled here is shown in a Venn diagram (Fig. 3C). 1,190 out of 1,738 domains ( $70 \%$ )

368 are shared widely such that $855(\sim 50 \%)$ are distributed across all the three major taxa and the rest

369 shared between two of the three taxa. 
A closer look at the core-genes datasets shows that the regions of the MSAs that are

371

372

373

374

375

376

377

378

379

380

381

382

383

384

385

386

387

388

389

390

391

392

393

394

395

396

397

398

399

400

retained after data filtering correspond to the distinct protein-domains (Fig. 3A; Table 2).

Genomic loci that can be aligned with high confidence using MSA algorithms are typically more conserved than those loci for which alignment uncertainty is high. Such ambiguously aligned regions of sequences are routinely trimmed off before phylogenetic analyses (Criscuolo \& Gribaldo 2010). Typically, the conserved well-aligned regions correspond to protein-domains with highly ordered 3D structures with specific 3D folds (Fig. 3B). Accordingly, the MSA in core-genes-I dataset corresponds to 35 distinct domains found in 29 genes (Table 2), while 50 distinct domains are found in 48 genes sampled in the core-genes-II dataset. In the core-genes-I dataset, the number of unique domains (or loci) sampled per species varies between 25 and 35, since not all loci are found in all species. While some loci are absent in some species, some loci are redundant. For instance, the P-loop NTP hydrolase domain, one of the most prevalent protein domains, is represented up to 9 times in many species (Table 2). Many central cellular functions are driven by the conformational changes in proteins induced by the hydrolysis of nucleoside triphosphate (NTP) catalyzed by the P-loop domain (Chothia et al. 2003).

Out of a total of 35 distinct domains in the core-genes-I dataset, 7 are redundant, with two or more copies represented per species. Similarly, 9 of the 50 domains have a redundant representation in the core-genes-II dataset (Table 2). The observed redundancy of the genomic loci in the MSA of core-genes is inconsistent with the common (and typically untested) assumption of using single-copy genes as a proxy for orthologous loci sampled for phylogenetic analysis. In contrast, the protein-domain datasets are composed of unique loci (Fig. 3C; Table 2). Further, the loci represented in the core-genes datasets make up only about $3 \%$ of the loci analyzed in SCOP-domain datasets in terms of the number of unique genomic loci sampled (Fig. 3D; Table 2).

Furthermore, regions of sequences that are filtered out, usually show higher variability in length, are less ordered and are known to accumulate insertion and deletion (indel) mutations at a higher frequency than in the regions that correspond to folded domains (Light et al. 2013; Wang et al. 2011). These variable, structurally disordered regions, which flank the structurally ordered domains, link different domains in multi-domain proteins (Fig. 3A). Multi-domain architecture (MDA), the N-to-C terminal sequence of domain arrangement, is distinct for a protein family, and differs in closely related protein families with similar functions (Fig. 3A). The variation in 
401 MDA also relates to alignment uncertainties. Taken together, there is a major loss of information

402 when core-genes datasets are employed for phylogenomic analyses compared to the protein403 domain datasets (Fig. 3C and Fig. 3D) Information loss is due to:

404 i. The small number of loci selected to start with in the core-genes datasets; at most 50 (Fig. 3D; Fig 1) compared to 1,738 in the SCOP-domain dataset (Fig. 3C; Fig. 2), and,

ii. The trimming of regions within MSAs due to alignment uncertainties (Fig. 3A).

Despite the relatively small number of characters that can be scored $(\sim 2,000)$, the protein domains datasets (Fig. 2) are more informative to resolve the major taxa than the core-genes datasets (Fig. 1), for which a large number of characters are scored $(\sim 10,000)$.

410

\section{Data Quality Affects Model Complexity Required to Explain Phylogenetic Datasets}

Resolving the monophyly or paraphyly of the Archaea is relevant to determining whether

413 the Three-Domains tree (Fig. 4A) or the Eocyte tree (Fig. 4B), respectively, is a better-supported 414 hypothesis. The Archaea are consistent with a monophyletic group in trees derived from a 415 relatively simpler, rate-homogeneous LG model applied to the core-genes-I dataset (Fig. 4A). 416 However, the archaea are consistent with a paraphyletic group in trees derived from the more 417 complex rate-heterogeneous versions of the LG model (Fig. 4B). In general, complex models tend to fit the data better. According to model selection tests for the core-genes-I dataset, the more complex versions of the LG model are better-fitting models than the simpler homogeneousLG model (Fig. 4C; Supplementary Table S2). Complex models account for various patterns of heterogeneity in amino acid substitutions. For instance, CSRH is accounted for by incorporating multiple rate-categories in the model. Substitution rate heterogeneity across different characters was approximated using a discrete Gamma distribution with 4,8 or 12 rate categories (LG+G4, $\mathrm{LG}+\mathrm{G} 8$ or $\mathrm{LG}+\mathrm{G} 12$, respectively). Model fit to data improves with the increase in complexity of the substitution model (Fig. 4C). Model complexity increases with any increase in the number of rate categories and/or the associated numbers of parameters that need to be estimated. Accordingly models that incorporate invariant sites $(\mathrm{LG}+\mathrm{GX}+\mathrm{I})$ or MSA-specific state frequencies $(\mathrm{LG}+\mathrm{GX}+\mathrm{F})$ and several combinations there of are even more complex. Recovering the Eocyte tree typically requires implementing complex models of sequence evolution rather than their relatively simpler (but over-simplified) versions (Williams \& Embley 2014). However, 
431 implementing more complex models did not change the tree topology (Fig. 4B) despite improved 432 model fit to data (Fig. 4C; Supplementary Table S2).

433 In contrast, trees inferred from the protein-domain datasets are consistent with

434 monophyly of the Archaea irrespective of the complexity of the underlying model, with respect 435 to CSRH (Fig. 4 D-F). The Mk model is the best-known probabilistic model of discrete character 436 evolution for complex characters coded as binary-state characters (Lewis 2001; Wright \& Hillis 437 2014). Since the Mk model assumes a stochastic process of evolution, it is able to estimate 438 multiple state changes along the same branch. Both a simpler rate-homogeneous version of the 439 Mk model (Fig. 4D), as well as more complex rate-heterogeneous versions with 4, 8 or 12 rate 440 categories $(\mathrm{Mk}+\mathrm{G} 4, \mathrm{Mk}+\mathrm{G} 8$ or $\mathrm{Mk}+\mathrm{G} 12$, respectively) recovered trees that are consistent with 441 the monophyly of the Archaea (Fig. 4E). The most complex model, Mk+G12 is the best fitting 442 model as seen from the LBF scores. A difference in LBF scores in the range of 3-5 is typically 443 considered strong evidence in favor of the better model and topological hypothesis; while LBF 444 difference of above 5 is considered very strong empirical evidence (Bergsten et al. 2013; Kass \& 445 Raftery 1995). The tree derived from the $\mathrm{Mk}+\mathrm{G} 4$ model is shown in Figure 4E. While the tree 446 derived from $\mathrm{Mk}+\mathrm{G} 8$ model is identical to the $\mathrm{Mk}+\mathrm{G} 4$ tree, the $\mathrm{Mk}+\mathrm{G} 12$ tree is almost identical 447 with minor differences within the bacterial groups (see Supplementary Figure S1). This is likely 448 to be due to the relatively more diverse set of species sampled from unclassified groups, and 449 hence a low-density coverage of taxonomic groups within the Bacteria. However, species 450 sampling from Archaea and Eukarya is relatively denser amongst taxonomic groups. of 0.99 while that of Bacteria and Eukarya is supported with a PP of 1.0 - in spite of substantially different fits of the model to the data. A notable exception to the sequence-based classification is that the traditional phylum Euryarchaeota is not supported in this tree. Paraphyly of Euryarchaeaota has also been observed with core-genes and single-gene datasets that were corrected for rate heterogeneity (Foster et al. 2009; Gouy et al. 2015). Nonetheless, the tree topology suggests that the Archaea is a distinct group. Even though the unrooted trees in Figure 4A and Figure 4D, E suggest monophyly of archaea, verification of the unique evolutionary history of archaea, or for that matter any other taxonomic group in the tree, depends on the placement of the root of the tree. Resolving the root of the global ToL is a difficult problem, both 
461 conceptually as well as technically, which is unlike other phylogenetic problems (see next 462 section).

463

464 Siblings and Cousins are Indistinguishable When Reversible Models are Employed

An unrooted tree derived from standard reversible evolution-models is oblivious to the root, and thus has no evolutionary direction (Fig. 5A, B). Therefore an unrooted (undirected) tree is uninformative about: (1) ancestor-descendant polarity of taxa; (2) branching order; (3) evolutionary groups (or clades); and (4) ancestral and derived states. Given that a primary objective of phylogenetic analyses is to identify clades and the relationships between these clades, it is not possible to interpret an unrooted tree meaningfully without rooting the tree (see Fig. 5). Identification of clades as well as inferences of relationships between clades depends on the placement of the root or on prior assumptions about the root. In general, it is not possible to make evolutionary inferences from any unrooted (undirected) topological hypothesis. For instance, although a DDN is useful to diagnose character conflicts in phylogenetic datasets and to postulate evolutionary hypotheses, a DDN by itself cannot be interpreted as an evolutionary network, because the edges do not necessarily represent evolutionary phenomena and the nodes do not represent ancestors (Huson \& Bryant 2006; Morrison 2009). Therefore, evolutionary relationships cannot be inferred from a DDN. Likewise, evolutionary relationships cannot be inferred from unrooted trees, even though nodes in an unrooted tree do represent ancestors and an evolution model defines the branches (Fig. 5A, B). An unrooted tree is consistent with more than one rooted tree (Fig. 5). ancestors as follows:

i. Any one of the inferred-ancestors at the resolved bipartitions (open circles in Fig. 5A, B), or

ii. Any one of the yet-to-be-inferred-ancestors that lies along the stem-branches of the unresolved polytomy (green stars Fig. 5A, B) or along the internal-braches. additional bipartition and an ancestor that is neither inferred from the source data nor deduced from the underlying character evolution model. Hence rooting, and interpreting the ToL depends on: 
492

493

494

495

496

497

498

499

500

501

502

503

504

505

506

507

508

509

510

511

512

513

514

515

516

517

518

519

520

521

522

i. Prior knowledge - e.g. fossils or known sister-group (outgroup) taxa, or

ii. Prior beliefs/expectations of the investigators - e.g. simple is primitive (Nasir \& Caetano-Anollés 2015; Whittaker 1969), bacteria are primitive (Sagan 1967; Stanier \& Niel 1962), archaea are primitive (Woese \& Fox 1977), etc.

Both of these options are independent of the data used to infer the unrooted ToL. Accordingly, both the Three-Domains hypothesis and the Eocyte hypothesis depend on the notion that the root should be placed on the stem branch leading to the bacteria (root R1 in Figure 5) in the unrooted tree. Other possible rootings and the resulting rooted-tree topologies are shown in Figure 5 C-J. In the unresolved tree (Fig. 4B and Fig. 5B) archaea would be paraphyletic irrespective of the placement of the root. In all other cases (Fig. 4A and Fig. 5B, C), if the root lies on any of the internal branches (e.g. R3 in Fig. 5A, B), or corresponds to one of the internal nodes within the archael radiation (e.g. R5 in Fig. 5A, B), the Archaea would not constitute a unique clade (Fig. 5). However, if the root lies on one of the stem branches (R1/R2/R4 in Fig. 5B), monophyly of the Archaea would be unambiguous (Fig. 5F, G, I). Determining the evolutionary relationship of the Archaea to other taxa, though, requires identifying the root.

The common practice of a posteriori rooting, i.e. converting an unrooted (undirected)

ToL into a rooted (directed) ToL, by hand, implies prior knowledge of the polarity of character transitions from ancestral-to-derived states. In other words, prior knowledge of the ancestral (root) states of characters is necessary to root a tree, which is commonly inferred from outgroup taxa. In the absence of prior knowledge of the root, directional evolution-models are useful for identifying the root (Huelsenbeck et al. 2002; Klopfstein et al. 2015; Yang \& Roberts 1995). Unlike reversible models, directional models are able to identify the polarity of state transitions, and thus the root of a tree. Moreover, directional models are useful to evaluate the empirical support for prior beliefs about the universal common ancestor (UCA) at the root of the ToL (Harish \& Kurland 2017a). Directional evolution refers to two distinct, but related aspects of the evolutionary process, nonreversibility and nonstationarity (Harish \& Kurland 2017b; Klopfstein et al. 2015). Nonreversibility refers to the asymmetric propensity of character transitions, i.e. propensity for change from one state to another along the tree is different from a change in the reverse direction. Nonstationarity refers to change in frequencies of characters (or states) in evolutionary time when conditions within a group differs from the conditions in its sister groups and thus at the root of the tree. Taken together, directional evolution refers to a nonrandom, and 
523 nonreversible shift in the marginal distribution of traits during evolutionary time (Klopfstein et 524 al. 2015).

525 The Bayesian model selection test (implemented in MrBayes) to detect directional trends 526 chooses the directional model (Fig. 6B), overwhelmingly, over the reversible model for the 527 SCOP-II dataset (Fig. 2B). Further, the best-supported rooting corresponds to root R4 (Fig. 5G 528 and Fig. 6A, C). Monophyly of the Archaea is maximally supported (PP of 1.0). Furthermore, 529 this rooting places Archaea sister to Bacteria with the highest support (PP 1.0). Accordingly, a 530 higher order taxon, Akaryotes, proposed earlier (Forterre 1992) forms a clade with robust 531 support (PP 1.0). Thus Akaryotes (or Akarya) and Eukarya are sister clades that diverge from the 532 UCA at the root of the ToL. Identical results were obtained for the SCOP-I dataset (Fig. 2A) as 533 reported previously (Harish \& Kurland 2017a). The placement of the root as well as the tree 534 topology is robust against long-branch attraction (LBA) artifacts due to CSRH and LSRH (Fig. $5356 \mathrm{C})$.

The simplest model, the standard stationary and reversible evolution-model, is the worst 537 fitting model far and away (Fig. 6C). As such, complex models that account for nonreversibility, 538 nonstationarity, CSRH and LSRH are the better fitting models. In all cases, and regardless of 539 model complexity, root R4 is the best-supported rooting (Fig. 5I). Thus the Two-Empires of life 540 hypothesis (Mayr 1998) is the best-supported ToL (Fig. 6A, C). Alternative rootings are much 541 less likely, and therefore other ToL hypotheses are not supported (Fig. 6C). Accordingly, 542 independent origin of eukaryotes as well akaryotes is the best-supported scenario. The Three543 domains ToL (root R1; Fig. 5F) is $10^{171}$ times less likely, and the scenario proposed by the 544 Eocyte ToL (root R1, Fig. 5C) is highly unlikely, as are other scenarios (root R2, R3; Fig. 5 G$545 \mathrm{H}$ ). The traditional belief that simple is primitive, as well as beliefs that archaea are primitive or 546 that archaea and bacteria evolved before eukaryotes, are not supported either.

\section{DISCUSSION}

549 Improving Data Quality Can Be More Effective for Resolving Recalcitrant Branches than 550 Increasing Model Complexity

A diversity of evolutionary signatures in molecular sequence data is utilized by different 552 analytical approaches to recover phylogenetic signal. Recovery of phylogenetic signal in 553 sequence-alignment data by the analysis of variation in single-residue substitutions is the 
554 predominant (standard) approach. Other less frequently used sources of phylogenetic signal

555 includes variation in triplet-codons, multiresidue indels in protein-coding and non-coding loci as

556 well as variation in the gain, loss and copy number of the loci per se (Harish \& Kurland 2017b;

557 Hillis 1999; Rokas \& Holland 2000). In the phylogenetic literature, the concept of data quality

558 refers to the quality or the strength of the phylogenetic signal that can be recovered from the

559 data. The strength of the phylogenetic signal is proportional to the confidence with which unique

560 state-transitions can be determined for a given set of characters on a given tree. Ideally,

561 historically unique character transitions that entail rare evolutionary innovations are desirable to

562 identify patterns of uniquely shared innovations (synapomorphies) among lineages.

563 Synapomorphies are the diagnostic features used for assessing lineage-specific inheritance of

564 evolutionary innovations. Therefore identifying character transitions that are likely to be low

565 probability events is crucial for the accuracy of phylogenetic analysis.

566 In their pioneering studies, Woese and colleagues identified unique features of the SSU

567 rRNA - "oligonucleotide signatures" - to determine evolutionary relationships (Woese 2004).

568 An underlying assumption was that the probability of occurrence of the same set of oligomer

569 signatures by chance, in non-homologous sequences, is low in a large molecule like SSU rRNA

570 (1500-2000 nucleotides). Further, the study found that only oligomers that were six nucleotides

571 or longer were robust markers of homology of the SSU rRNA. Oligomers shorter than six

572 nucleotides were statistically less likely to be efficient markers of homology (Pechman \& Woese

573 1972; Woese et al. 1975). Thus larger oligomer signatures are more robust characters than

574 shorter oligomers or monomers to determine a phylogeny of the SSU rRNA gene (or gene

575 family).

$576 \quad$ However, as sequencing of full-length rRNAs and statistical models of nucleotide

577 substitution became common, complex oligomer-characters were replaced by elementary

578 nucleotide-characters; and more recently by amino acid characters (Fig. 1). Identifying rare or

579 historically unique substitutions in empirical datasets has proven to be difficult (Parker et al.

580 2013; Rokas \& Carroll 2008), consequently the uncertainty of resolving the deeper branches of

581 the ToL using marker gene-sequences remains high. A primary reason is the relatively higher

582 prevalence of phylogenetic noise (homoplasy) in primary sequence datasets (Fig. 1), due to the

583 characteristic redundancy of nucleotide and amino acid substitutions and the resulting difficulty

584 in distinguishing phylogenetic noise from signal (homology) (Philippe et al. 2011; Rokas \& 
585 Carroll 2006). Better-fitting (or best-fitting) models are expected to recover phylogenetic signal 586 more efficiently and thus explain the data better, but tend to be more complex than worse fitting 587 models (Lartillot \& Philippe 2004; Williams \& Embley 2014).

588 Unrooted topologies estimated from the core-genes-I dataset using CSRH-LG models are 589 congruent (Fig 4B), in spite of the significantly different model-fits to the data (Fig. 4C).

590 Likewise, both unrooted- (Fig. 4D, E) and rooted (Fig. 6A) topologies estimated from the SCOP-

591 II dataset using $\sim 15$ distinct models of increasing complexity, and significantly different model-

592 fits to data (Fig. 4E and Fig 6C), are largely congruent. These results show that increased model 593 complexity, or improved model-fit to data, does not necessarily resolve conflicting signals in 594 phylogenetic datasets. Although increasing model complexity can correct errors of estimation 595 and improve the fit of the data to the tree, it is not a solution to improve phylogenetic signal, 596 especially when the historical signal is exceedingly limited or absent in the source data (Fig. 1 597 and Fig. 3C, D; Table 2).

598 The idea of 'oligonucleotide signatures' used for estimating a gene phylogeny has been 599 extended, naturally, to infer a genome phylogeny (Graham et al. 2000). The signatures were 600 defined in terms of protein-coding genes that were shared among the Archaea. However, as 601 proteins are recombinant-mosaics of domains, domains are unique genomic signatures (Fig. 3).

602 Protein-domains identified by SCOP correspond to complex 'multidimensional signatures' 603 defined by: (i) a unique 3D fold, (ii) a distinct sequence profile, and (iii) a characteristic function.

604 Though domain recombination is frequent, substitution of one protein domain for another has not 605 been observed in homologous proteins (Fig. 3). Thus, protein-domains are "functional genomic 606 signatures". For phylogenomic applications, protein domains are 'sequence signatures' that 607 essentially correspond to single-copy orthologous loci when coded as binary-state (presence608 absence) characters. These sequence signatures are consistent with unique, non-recombining 609 genomic loci, and are identified using sophisticated statistical models - profile hidden Markov 610 models (pHMMs) (Eddy 1998; Park et al. 1998) - that can be used routinely to annotate and 611 curate genome sequences in automated pipelines (Fang et al. 2013; Gough et al. 2001).

612 For these reasons, protein-domains are highly effective phylogenetic markers for which

613 character-homology can be validated through more than one property: statistically significant (i)

614 sequence similarity, (ii) 3D structure similarity, and (iii) function similarity. In addition,

615 employing loci for protein-domains maximizes the genomic information that can be employed 
616 for phylogenetic analysis (Table 2; Fig. 3C, D). Even though many other genomic features are

617 known to be useful markers (Rokas \& Holland 2000), protein domains are the most conserved as

618 well as most widely applicable genomic characters (Fig. 3C). Protein-domain characters are not

619 without caveats (see (Harish \& Kurland 2017b) for a discussion).

620 Character recoding is found to be effective in reducing the phylogenetic noise in primary

621 sequence data (Susko \& Roger 2007). This is a form of data simplification wherein the number

622 of amino acid alphabets is reduced to a smaller set of alphabets (usually from 20 to 6) that are

623 frequently substituted for each other. Character recoding into reduced alphabets is useful to

624 minimize phylogenetic artifacts such as LBA due to substitution saturation or compositional

625 heterogeneity. However, character recoding does not reduce the noise in the core-genes-II dataset

626 (Fig. 2D). Contrary to the expectation, there is an increase in the apparent noise, as seen from an

627 increase in the extent of reticulation compared to the original (untreated) data (Fig. 2C).

628 Common methods of estimating mutational saturation in sequences, particularly from multi-gene

629 concatenations tend to underestimate the degree of saturation (Whelan et al. 2015). This seems to

630 be the case especially for ribosomal proteins, which dominate the core-genes datasets (see

631 Whelan et al (2015) for a detailed characterization).

632 Therefore, datasets in which phylogenetic noise is inherently limited are more desirable,

633 to minimize ambiguities. Like amino acids, protein-domains are also modular alphabets, albeit

634 higher order and more complex alphabets of proteins. Moreover, unlike the 20 standard amino

635 acids, there are approximately 2,000 unique protein domains identified at present according to

636 SCOP (Murzin et al. 1995). The number is expected to increase; theoretical estimates range

637 between 4,000 and 10,000 distinct domain modules, depending on the classification scheme

638 (Govindarajan et al. 1999). Coding homologous features as binary characters is the simplest

639 possible representation of data for describing historically unique events. Accordingly, resolving

640 character conflicts observed in the data (Fig. 2) would be less demanding, as such conceptually,

641 and also computationally less expensive for large-scale empirical datasets.

642

643 Sorting Evolutionary Signal from Noise

644 Single-copy genes are employed as phylogenetic markers to minimize phylogenetic noise

645 caused by reticulate evolution including hybridization, introgression, recombination, horizontal

646 transfer (HT), duplication-loss (DL), or incomplete lineage sorting (ILS) of genomic loci. 
647 However, the noise observed in the DDNs based on MSA of core-genes (Fig. 1) cannot be 648 directly related to any of the above genome-scale reticulations, since the characters are individual 649 nucleotides or amino acids. Apart from stochastic character conflicts, the observed conflicts are 650 better explained by convergent substitutions, given the redundancy of substitutions. Convergent 651 substitutions caused either due to stringent selection or by chance are a well-recognized form of 652 homoplasy in gene-sequence data (Castoe et al. 2010; Philippe et al. 2011; Rokas \& Carroll 653 2008), and based on recent genome-scale analyses it is now known to be rampant (Foote et al. 654 2015; Liu et al. 2010).

655 The observed noise in the DDNs based on protein-domain characters (Fig. 2), however, 656 can be related directly to genome-scale reticulation processes and homoplasies. In general, 657 homoplasy implies evolutionary convergence, parallelism or character reversals caused by 658 multiple processes. In contrast, homology implies only one process: inheritance of traits that 659 evolved in the common ancestor and were passed to its descendants. Operationally, tree-based 660 assessment of homology requires tracing the phylogenetic continuity of characters (and states), 661 whereas homoplasy manifests as discontinuities along the tree. Since clades are diagnosed on the 662 basis of shared innovations (synapomorphies) and defined by ancestry accuracy of a phylogeny 663 depends on an accurate assessment of homology (Avise \& Robinson 2008; Hennig 1965; 664 Morrison 2006; Padian et al. 1994)

665 Identifying homoplasies caused by character reversals, i.e. reversal to ancestral states 666 requires identification of the ancestral state of the characters under study. However, 667 implementing reversible models precludes the estimation of ancestral states, in the absence of 668 sister groups (outgroups) or other external references. Thus, the crucial distinction between 669 similarity due to homoplasy and homology as well as between shared ancestral homology 670 (symplesiomorphy) and shared derived homology (synapomorphy) is not possible with unrooted 671 trees derived from standard reversible models. Hence, unrooted trees (Fig. 4) are not

672 evolutionary trees per se, as they are uninformative about the evolutionary polarity (Morrison 673 2006; Wiley \& Lieberman 2011). Thus, identifying the root (or root-state) is crucial to (i) 674 determine the polarity of state transitions, (ii) identify synapomorphies, and (iii) diagnose clades. 675 For complex characters such as protein-domains, character homology can be determined 676 with high confidence using sophisticated statistical models (HMMs). Homology of a protein 677 domain implies that the de novo evolution of a genomic locus corresponding to that protein 
678 domain is a unique historical event. Therefore, homoplasy due to convergences and parallelisms 679 is highly improbable (Mackin et al. 2014; Pethica et al. 2012). Although a handful of cases of 680 convergent evolution of 3D structures are known, these instances relate to relatively simple 3D 681 folds coded for by relatively simple sequence repeats (Mistry et al. 2013). However, the vast 682 majority of domains identified by SCOP correspond to polypeptides that are on average 200 683 residues long with unique sequence profiles (Gough et al. 2001; Pethica et al. 2012). Thus, 684 identifying homoplasy in the protein-domain datasets depends largely on estimating reversals, 685 which will be cases of secondary gains and losses. For instance, reversals due to gain-loss-regain 686 events caused by HT or DL-HT are homoplasies. Such secondary gains are more likely to relate to HT events than to convergent evolution, for reasons specified above. Instances of reversals are minimal, as seen from the strong directional trends detected in the data (Fig. 6B). Thus, employing complex molecular characters minimizes uncertainty in determining polarity of state transitions, identifying synapomorphies and diagnosing clades. evolutionary novelties, the discovery of clades is fundamental for describing and diagnosing sister group differences (Sanderson 2005). A well-recognized deficiency of phylogenetic inference based on primary sequences is the abstraction of evolutionary 'information': For instance, 'information' relevant to diagnosing clades and support for clades is abstracted to branch lengths. Branch-length estimation is, ideally, a function of the source data and the underlying model. However, in the core-genes-I dataset the estimated branch lengths and the resulting tree is an expression of the model rather than of the data (Fig. 4A, B). Some pertinent questions then are: should diagnosis of clades and the features by which clades are identified be restricted to substitution mutations in a small set of loci and substitution models? Are substitution mutations in 40-50 loci more informative, or the evolution of unique genomic locifunctional genomic signatures - more informative?

Proponents of the total evidence approach recommend that all relevant information molecular, biochemical, anatomical, morphological, fossils - should be used to reconstruct evolutionary history, yet genome sequences are the most widely applicable data at present (Rokas \& Holland 2000; Wheeler et al. 2013). Accordingly, phylogenetic classification is, in

707 practice, a classification of genomes. There is no a priori theoretical reason that phylogenetic 708 inference, especially of the global ToL, should be restricted to a small set of genomic loci 
709 corresponding to the core-genes, nor is there a reason for limiting phylogenetic models to

710 interpreting patterns of substitution mutations alone. The ease of sequencing and the practical

711 convenience of assembling large character matrices, by themselves, are no longer compelling

712 reasons to adhere to the traditional marker gene-sequence analysis.

713 When phylogenetic inference is based on the protein-domain datasets, the gain and loss

714 of distinct sets of "functional signatures" that define clades can be identified, which is unlike

715 inferences based on core-genes datasets (see Supplementary Information in Harish et al (2013)).

716 Annotations for reference genomes of homologous protein-domains identified by SCOP and

717 other protein classification schemes, as well as tools for identifying corresponding sequence

718 signatures, are readily available in public databases. An added advantage is that the biochemical

719 function and molecular phenotype of the domains are readily accessible as well, through

720 additional resources including the protein data bank (PDB) and InterPro (Finn et al. 2016).

721

722 The Critical Distinction Between Gene-centered and Genome-centered Phylogenetic

723 Models

724 As mentioned in the previous section, assessment of homology is fundamental for

725 inferring character evolution as well as evolutionary relationships between the operational

726 taxonomic units (OTUs). Because OTUs are defined arbitrarily, the distinction between gene-

727 OTUs and genome-OTUs cannot be emphasized enough. This distinction is crucial for the

728 assessment of molecular homology, since homology is a hierarchical concept (Dickinson 1995;

729 Morrison et al. 2015). Homology at one level in the hierarchy need not necessarily imply

730 homology at another level of biological organization. Accordingly, homology at different levels

731 is detected using different criteria. For instance, homology of gene-OTUs in any given gene

732 cluster is inferred from statistically significant overall-similarity of the genes without considering

733 the homology of individual nucleotide or amino-acid characters. Clusters of gene-OTUs are

734 identified and classified into families based on measures of overall-similarity, which is estimated

735 either as pairwise sequence similarity (e.g. BLAST) or similarity to sequence profiles (e.g. PSI-

736 BLAST and HMM) (Pearson \& Sierk 2005).

737 Measures of overall similarity do not distinguish between homologous similarity and

738 similarity by chance (homoplasy) of individual characters. In contrast, phylogenetic methods

739 have the distinct advantage of distinguishing the evolutionary signal of homology from the noise 
740 due to homoplasy (Avise \& Robinson 2008; Morrison 2006). Even though determining the

741 evolutionary polarity of character-transitions is key to distinguish signal from noise, many

742 commonly used tools of inference such as MSAs and unrooted trees are oblivious to the polarity

743 of evolutionary transitions, and hence to the evolutionary path. This can often result in erroneous

744 estimates and spurious placements of OTUs (Eisen 1998; Kurland et al. 2003). That is to say that

745 the nearest neighbor in an unrooted tree (or in an overall-similarity network) need not necessarily

746 be the closest relative, as shown in Figure 5. Identifying the root is critical even when the OTUs

747 are individual genes to avoid misleading conclusions. This is decidedly relevant to phylogenomic

748 analyses designed to identify clades and to determine trends in macroevolution (Harish \&

749 Kurland 2017b; Harish \& Kurland 2017c).

750 It is becoming increasingly clear that conventional phylogenomic approaches frequently

751 fail to resolve the deeper nodes of the ToL reliably (Philippe et al. 2011; Shen et al. 2017;

752 Whelan et al. 2015). Existing methods that rely on recovering phylogenetic signal from MSAs

753 neither identify, nor describe sister-group differences satisfactorily in spite of employing several

754 hundreds of MSAs. A well-studied problem is the identification of the root of the Metazoa

755 (animals), which is a relatively shallow node in the global ToL compared to the root of the

756 Archaea. Efforts to resolve the metazoan-root have employed concatenated MSAs of up to 1,000

757 genes, and yet it remains ambiguous (Philippe et al. 2011; Shen et al. 2017; Whelan et al. 2015).

758 In comparison to the metazoan-root, the number of genes that can be aligned to the resolve the

759 root of the global ToL is extremely limited, to approximately 50 (Zaremba-Niedzwiedzka et al.

760 2017).

761 One approach to overcome this limitation involves estimating trees of individual gene-

762 families en masse. Topologies of individual gene-OTUs are summarized in order to estimate the

763 support for the monophyly (unique origin) of major taxa: Archaea, Bacteria and Eukarya

764 (Nelson-Sathi et al. 2015; Rochette et al. 2014; Thiergart et al. 2012). However, all of the trees

765 are derived from reversible and stationary models, which yield unrooted trees. Therefore $a d$ hoc

766 sister groups (outgroups) are specified to determine the root and polarity of evolution. And, the

767 choice of sister group(s) is itself based on measures of overall similarity. In other words the

768 identification of the origin (root) of any given gene family is ad hoc (Fig. 5A, B).

769 Misidentification of sister groups along with spurious placements of gene-OTUs can potentially

770 confound the interpretation of such unrooted trees (Graham et al. 2002). 

paralogous pair (Baldauf et al. 1996; Iwabe et al. 1989) is known to be error-prone and highly ambiguous, due to systematic errors including LBA, compositional bias and model

774

775

776

777

778

779

780

781

782

783

784

785

786

787

788

789

790

791

792

793

794

795

796

797

798

799

800

801

misspecification (Brinkmann \& Philippe 1999; Gouy et al. 2015). Remarkably, sequences corresponding to only one of the two conserved domains common to EF-Tu and EF-G, the Ploop-containing NTP hydrolase domain (Fig. 3A) can be aligned with confidence. This singledomain MSA is $\sim 200$ residues in length. Implementing better-fitting substitution models results in two alternative rootings (Gouy et al. 2015). These are root R1 (on the branch leading to bacteria) and a root within the archaea that is similar to root R5 (Fig. 5B). These alternative rootings relate to distinct, irreconcilable scenarios. Further, the EF-Tu-EF-G paralogous pair is only 2 of 57 known paralogs of the translational GTPase protein superfamily (Atkinson 2015). Thus the assumption that EF-Tu-EF-G duplication is a unique event, which is essential for the paralogous outgroup-rooting method, is untenable (Fig. 3). Furthermore, the root inferred for one gene (or domain) family may not be applicable to another family due to the prevalent discordance between individual gene trees, and between gene trees and species trees. Therefore, the Dayhoff duplicate-gene-rooting method (Schwartz \& Dayhoff 1978; Woese et al. 1990) is not suitable to root genome trees or species trees.

These findings underscore the importance of acknowledging the hierarchical difference between genome-OTUs and gene-OTUs as well as relevant character evolution models used to determine evolutionary relationships (Boussau \& Daubin 2010; Coenye et al. 2005; Harish \& Kurland 2017b). However, current phylogenomic approaches involve analysis of a concatenatedMSA of highly conserved (core) genes on the one hand and independent analysis of less conserved (accessory) genes on the other. That is, contrasting approaches are applied to different regions of genomes that are conserved to different extents. Such contrasting treatments point to a rather obvious predicament. That is, if it is not possible recover reliable phylogenetic signal from concatenated-MSAs, of many conserved marker genes (Fig. 1), how reliable is the signal from individual MSAs of relatively less conserved gene families? Not quite reliable, evidently, since existing MSA-analysis methods are unable to adequately distinguish phylogenetic signal and noise for the OTUs employed.

In other words, existing phylogenomic methods that employ reversible/stationary sequence evolution-models are suboptimal for determining the temporal order of key 
802 evolutionary transitions in the ToL. Therefore, inferences of the origin of individual gene

803 families as well as the estimated evolutionary path are likely to be error prone. For instance, it is

804 not possible to determine if anomalous placement of a gene-OTU is due to a lack of phylogenetic

805 signal or HT without adequately distinguishing signal from noise (Rochette et al. 2014).

806 Accordingly, the origin of individual gene families may be untraceable from the analysis of

807 single-gene MSAs using existing methods. This calls for an explicit distinction between

808 evolutionary inferences drawn from qualitatively different gene-scale (Fig. 1) and genome-scale

809 (Fig. 2) evolutionary signatures as well as qualitatively different (e.g. directional vs reversible)

810 evolution models (see next section).

811

812 Untangling Data Bias, Model Bias and Interpretation Bias (Prior Beliefs)

813 A single class of genes, those encoding ribosomal proteins (RPs) dominates core-genes

814 datasets; for example RPs make up 66\% (32/48) of the core-genes-II dataset (Zaremba-

815 Niedzwiedzka et al. 2017). Further, core-genes datasets predominantly relate to one functional

816 class (Translation) of the $\sim 25$ functional classes assigned to clusters of orthologous groups

817 (COGs) (Tatusov et al. 2000). In contrast, the SCOP-domain datasets span all functional classes

818 that can be assigned to homologous sequences. Further, the monophyly of Archaea, and the

819 placement of Archaea sister to Bacteria are supported by the highest posterior probability of 1.0

820 (Fig. 6). The results are robust to stochastic errors as well as to potential systematic errors related

821 to rate heterogeneity, both CSRH and LSRH. Furthermore, unlike primary sequence data in

822 which compositional bias is a potential source of systematic error, the distinct genomic

823 compositions of unique SCOP-domains are informative about relationships among taxa (Fang et

824 al. 2013; Harish \& Kurland 2017a). Importantly the use of unique, complex molecular

825 characters, along with directional evolution-models enable the assessment of relationships that

826 extend beyond the phylogeny of a specific group for which suitable outgroups are unavailable. It

827 is also useful in cases where the choice of outgroup sequences is restricted and/or prone to

828 artifacts (e.g. LBA and compositional bias), which can not only confound the placement of the

829 root, but can also influence the ingroup phylogeny (Graham et al. 2002).

830 Moreover, systematic errors in phylogenetic inference (e.g. LBA or model

831 misspecification) are primarily errors in adequately distinguishing homologous similarities from

832 homoplastic similarities (Avise \& Robinson 2008; Morrison 2006; Philippe et al. 2011). 
833 Homologies, synapomorphies and homoplasies are qualitative inferences, yet are inherently

834 statistical. The probabilistic framework has proven to be powerful for testing alternative

835 hypotheses. Log odds ratios, such as LLR and LBF, are measures of how one changes belief in a

836 hypothesis in light of new evidence (Huelsenbeck et al. 2004). Accordingly, directional

837 evolution-models are the most optimal explanations of the observed distribution of genomic

838 signatures (Figs. 3 and 6). Such directional trends overwhelmingly support the monophyly of the

839 Archaea, as well as the sisterhood of the Archaea and the Bacteria, i.e. monophyly of Akarya as

840 well as monophyly of Eukarya (Fig 6).

841 These findings are in stark contrast compared to those of MSA-based analyses (Nelson-

842 Sathi et al. 2015; Rochette et al. 2014; Thiergart et al. 2012; Zaremba-Niedzwiedzka et al. 2017).

843 Since these contrasting results cannot be reconciled, it is worthwhile to revisit the source data

844 that support the conflicting hypotheses. As mentioned earlier, the DDNs derived from single-

845 gene and core-genes data (Fig. 1) as well as from protein-domain data (Fig. 2) are both

846 quantitatively and qualitatively different representations of genomes (Fig. 3). Accordingly,

847 models that describe qualitatively different processes of molecular evolution are required to

848 explain the data. The relevant evolutionary processes/events are mutually exclusive: While the

849 former is explained by point mutations within the selected loci (Fig. 1), the latter is explained by

850 gain and loss (or birth and death) of the selected loci (Fig. 2). Likewise, the sources of the

851 observed conflicts in the DDNs are qualitatively different as well. The sources of the observed

852 conflicts, though, are unknown a priori in both cases (Fig. 1 and Fig. 2).

853 In primary sequence data (Fig. 1) conflicts could arise due to stochastic errors (e.g.

854 gene/site sampling and alignment errors) and evolutionary processes (e.g. mutational bias due to

855 uneven damage and repair of genes). Conflicts in the protein-domain datasets (Fig. 2) could arise

856 due to genome/locus sampling errors and evolutionary processes such as ILS or some type of

857 gene flow, including HT. A naïve interpretation of both the DDNs (Figs. 1 and 2) that discounts

858 the complexity of the conflicts would be to assume that all the reticulations represent HT events.

859 Likewise, another naïve interpretation of the marker genes-DDNs (Fig. 1) that disregards the

860 evolutionary polarity is - the archaea appear to be a chimeric group derived from a fusion of

861 bacterial and eukaryote lineages, and that the members of the group diverged following a fusion

862 event (Fig. 1 A-D). 
864 standard phylogenetic theory, provided that the polarity of character transitions can be

865 determined. It is more so for unique phylogenetic characters such as protein-domains, as

866 described earlier. However, it is nontrivial to distinguish between random noise and phylogenetic

867 noise (homoplasy) on the one hand, and between the different causes of phylogenetic noise

868 (Avise \& Robinson 2008; Morrison 2009). Existing methods do not distinguish between the

869 different types of noise and hence it is hardly quantified as such. Therefore, there is a tendency to

870 interpret the observed conflicts as evolutionary events (i.e. prior belief), most often as HT events.

871 This is especially the case when inferences are drawn from analyses of single-gene MSAs

872 (Murray et al. 2016). As emphasized earlier, unrooted trees as such do not distinguish between

873 phylogenetic signal and noise, let alone distinguishing between the different types of noise. As a

874 result, identification of 'close relatives' or HT based on unrooted trees and other measures of

875 overall similarities in single-gene MSAs may not be optimal, especially when the signal to noise

876 ratio is low in a given MSA (Eisen 2000; Salzberg 2017).

877 Inference of historical HT events is, by necessity, statistical as is any other unobservable

878 event from the evolutionary past (Salzberg et al. 2001). Statistical inferences are as such robust

879 when a large number of features can be compared. As far as the global ToL is concerned, MSAs

880 of individual genes are not sufficiently large on their own given the data quality (Figs. $1 \& 3$ ).

881 Conflicting tree topologies are, by and large, associated with rate- and compositional

882 heterogeneities in both concatenated and single gene analyses (Gouy et al. 2015; Williams \&

883 Embley 2014). Several models that account for (correct for) errors in measuring rate- and

884 compositional heterogeneities in MSAs have been developed (Arenas 2015; Gouy et al. 2015).

885 However, conflicts in tree topologies that arise due to other systematic errors, such as the

886 prevalent assumptions of reversibility and stationarity of the evolutionary process, are rarely

887 acknowledged. This is highly relevant, and crucial for rooting trees and for inferring gene origin

888 and HT events, especially at the grand scale of the ToL.

889 Indeed, careful measurements of HT show a strong correlation between potential HT

890 events and systematic error in MSA-based estimates. Most potential HT events inferred from

891 anomalous placements of gene-OTUs are associated with systematic error, even among closely

892 related lineages - e.g. within a single genus (Murray et al. 2016). These findings suggest that

893 reversible substitution models and unrooted gene trees are suboptimal tools to diagnose 'gains' 
894 of genomic loci by HT. Estimation of gains is better suited for methods that are designed to 895 systematically model gains and losses in genomes (Klopfstein et al. 2015; Zamani-Dahaj et al.

896 2016). In general, systematic models of gene (or domain) gain-and-loss estimate significantly

897 lower frequencies of HT (Zamani-Dahaj et al. 2016), compared to HT estimates based on overall

898 similarities (Nelson-Sathi et al. 2015; Roettger et al. 2009). HT estimates are consistently lower 899 across the ToL: within Rickettsia, a genus (Murray et al. 2016); within Cyanobacteria, a phylum 900 and within Archaea (Zamani-Dahaj et al. 2016). In addition, and importantly, these studies show 901 that the tree-like pattern of inheritance of genomic loci is explained largely by the variation in 902 rates of loss among lineages, and that the fraction of loci that are prone to HT is a minority 903 (Harish et al. 2013; Zamani-Dahaj et al. 2016).

904 These findings are incompatible with the conventional view that extensive historical 905 horizontal transfer has resulted in mosaic genomes in extant species of archaea (Nelson-Sathi et 906 al. 2015) bacteria (Lake et al. 1999; Martin 1999) and eukaryotes (Rochette et al. 2014; Thiergart 907 et al. 2012). This incongruence is unsurprising for the simple reason that these incompatible 908 inferences are drawn from qualitatively different evolution-models that describe mutually exclusive processes of character evolution. It will be useful to recall that substitution mutations

910 in genomic loci (Fig. 1) and gain-loss (birth-death) of loci (Fig. 2) are mutually exclusive. These

911 issues are discussed extensively elsewhere (e.g. (Harish \& Kurland 2017a; Harish \& Kurland

912 2017c; Murray et al. 2016; Zamani-Dahaj et al. 2016).

913 Sophisticated statistical tests for evaluating tree robustness, and for selecting character-

914 evolution models are becoming a standard feature of phylogenetic software. However tests for

915 character evaluation are not common even though data quality is at least as important as the

916 evolution models that are posited to explain the data. Routines for collecting and curating data

917 upstream of phylogenetic analyses are rather eclectic. Besides, it is an open question as to 918 whether qualitatively different datasets (as in Fig.1 and Fig.2) can be compared effectively.

919 Nevertheless, employing DDNs and other tools of exploratory data analysis would be valuable to

920 identify conflicts that arise due to data collection and/or curation errors. In addition, it is

921 important to recognize the difference between DDNs (undirected networks) and evolutionary

922 networks (directed networks that represent evolutionary history) - just as it is important to

923 distinguish an unrooted tree from a rooted tree (Morrison 2006; Morrison 2009), to draw

924 evolutionary inferences (Fig. 5). 
926

927

928

929

930

931

932

933

934

935

936

937

938

939

940

941

942

943

944

945

946

947

948

949

950

951

952

953

954

\section{Additional Thoughts on Rooting the ToL}

Phylogenetic theory as well as related methods of discrete character analysis that were developed for the systematic classification of organismal families (Darwin 1859; Hennig 1965), was embraced, although not entirely, to determine the evolution and classification of gene families (Woese \& Fox 1977; Zuckerkandl \& Pauling 1965). The initial recognition of the Archaea was based on the comparative analysis of a single-gene (rRNA) family. It is remarkable that the uniqueness of the Archaea was identified by the comparative analyses of oligonucleotide signatures in a single-gene dataset (Woese \& Fox 1977). However the same is not true of the phylogenetic classification of Archaea, based on marker genes and reversible evolution-models. In spite of the large number of characters that can be analyzed, neither the rRNA genes nor multi-gene concatenations of core-genes have proven to be efficient markers to reliably resolve the phylogenetic affinities of the Archaea (Gribaldo et al. 2010; Gupta 2016). Consequently, there is a growing consensus that genomes as OTUs (Fig. 2), rather than genes as OTUs (Fig. 1), are not only more informative but are also more appropriate for organizing biodiversity, and for understanding the evolutionary history of species (Boussau \& Daubin 2010; Coenye et al. 2005; Harish \& Kurland 2017a).

Standard evolution-models implemented for phylogenomic analyses are limited to modeling variation in patterns of point mutations. These evolution-models are intimately linked to highly idealized concepts of molecular evolution, such as the universal molecular clock (Zuckerkandl \& Pauling 1965), the universal chronometer (Woese 1987), paralogous outgroup rooting (Schwartz \& Dayhoff 1978), etc., which are gene-centric concepts that were developed to study the gene, during the age of the gene. Moreover, these idealized notions originated from the analyses of relatively small single-gene datasets. Conventional phylogenomics of multi-locus datasets is a direct extension of the concepts and methods developed for single-locus datasets (Philippe et al. 2011). These methods rely exclusively on substitution mutations, which may not be ideal phylogenetic markers (Rokas \& Holland 2000). In contrast, the fundamental concepts of phylogenetic theory: homology, synapomorphy, homoplasy, character polarity, etc., even if idealized, are more generally applicable. And, apparently they are better suited for unique and complex molecular characters rather than for redundant, elementary sequence characters; with 
955 regards to determining both qualitative as well as statistical consistency of the data and the 956 underlying assumptions.

957 In the absence of prior knowledge of outgroups or of fossils, rooting the global ToL is

958 arguably one of the most difficult phylogenetic problems. The conventional practice of $a$

959 posteriori rooting, wherein an unrooted tree is converted into a rooted tree by adding an $a d$ hoc

960 root, encourages a subjective interpretation of the ToL. For example, the so-called bacterial

961 rooting of the ToL (root R1; Fig. 5) is the preferred rooting hypothesis to interpret the ToL even

962 though that rooting is not well supported (Gouy et al. 2015). Incorrect rooting may lead to

963 profoundly misleading conclusions about evolutionary scenarios and phylogenetic relationships,

964 and it appears to be common in phylogenetic studies (Graham et al. 2002). For example, root

965 placement between eukaryotes and akaryotes is incompatible with the chimeric origins of

966 eukaryotes (Harish \& Kurland 2017c; Harish et al. 2013; Kurland \& Andersson 2000).

967 Likewise, because of the central role of phylogenetic inference in biological

968 classification, incorrect rooting or accommodating a priori scenarios (e.g. endosymbiosis or

969 fusion scenarios for eukaryote origins) could confound systematic classification (Gribaldo \&

970 Brochier-Armanet 2012); for example, proposals for primary Kingdoms (Whittaker 1969; Woese

971 \& Fox 1977), primary Domains/Empires (Harish \& Kurland 2017a; Harish et al. 2013; Lake

972 1986; Mayr 1998; Williams et al. 2013; Woese et al. 1990) and other recent proposals for

973 systematic ranks such as Superphylum (Fuerst 2013; Guy \& Ettema 2011). Genomic signatures

974 and phylogenetic models that assess the polarity of evolutionary transitions will be valuable to

975 resolve conflicting proposals.

976

977 Conclusions and Future Directions

978 The Three-Domains of life hypothesis (Woese et al. 1990) was initially based on the 979 interpretation of an unrooted rRNA tree (of life) (Woese 1987; Woese \& Fox 1977). It was put

980 forward largely to emphasize the uniqueness of the Archaea, ascribed to an exclusive lineal

981 descent. The robust support for monophyly of the Archaea based on phylogenetic analysis of

982 genomic signatures agrees with other lines of evidence, molecular or otherwise (Garrett 1985;

983 Valentine 2007). Idiosyncratic features that support the uniqueness of the Archaea include the

984 subunit composition of supramolecular complexes like the ribosome, DNA- and RNA-

985 polymerases, biochemical composition of cell membranes, cell walls, and physiological 
986 adaptations to energy-starved environments, among other things. However, phylogenetic models

987 of the evolution of genomic signatures support a Two-Domains, or rather Two-Empires of Life

988 hypothesis (Mayr 1998). Neither the alternative Two-Domains/Eocyte hypothesis (Lake 1986)

989 nor the Three-Domains hypothesis (Woese et al. 1990) is supported. Accordingly, genomic

990 evolutionary signatures do not support the presumed primitive state of Archaea and Bacteria

991 (akaryotes), and the traditional belief that Archaea and Bacteria should be ancestors of Eukarya

992 (Sagan 1967; Spang et al. 2015; Williams et al. 2013; Woese \& Fox 1977; Zaremba-

993 Niedzwiedzka et al. 2017). The independent origins and parallel descent of eukaryote and

994 akaryote species (Gouy et al. 2015; Harish et al. 2013) is the best-supported hypothesis.

995 This study shows that phylogenetic inference based on functional genomic signatures and

996 directional evolution-models is less prone to systematic errors due to LBA, CSRH, LSRH and

997 compositional biases that often mislead MSA-based inferences. Consequently uncertainties in

998 resolving the branches of the ToL, especially the early divergences, can be minimized

999 effectively. The shortcomings of MSAs and substitution models can be overcome by employing

1000 complex molecular characters, which initially were thought to be a complementary set of

1001 phylogenetic markers that are useful for resolving difficult systematic problems (Rokas \&

1002 Holland 2000). However, given the qualitative differences of the data types, should MSA-based

1003 phylogenetic inferences be supplemented with complex molecular characters and corresponding

1004 character evolution-models? Or perhaps supplanted? I argue for the latter based on the findings

1005 of this study, and the limited perspective that is provided by the core-genes datasets towards

1006 understanding the early diversification of the ToL. The resolving power of gene-sequences using

1007 substitution models has been overstated - if not in general, it is evidently the case with regards to

1008 resolving the early diversification of archaea and the placement of the root of the global ToL.

1009 Employing genomic signatures is particularly relevant to study the evolution of the biodiversity

1010 of uncultivable microbial species that is characterized by genome sequences.

1011 It is worth emphasizing that the impact of LSRH (heterotachy) was not assessed in almost

1012 all recent studies that characterized incongruences in various phylogenomic datasets, including

1013 those of core-genes datasets. It appears that accounting for LSRH is unlikely to improve the

1014 analyses of core-genes datasets, though, it is a potential source of systematic error for the larger

1015 datasets such as those used to resolve the root of the metazoan-ToL. Perhaps, a stronger potential

1016 for systematic error is the assumption of reversibility and stationarity in standard evolution- 
1017 models. Both assumptions are made for mathematical simplicity and computational convenience,

1018 but may not be biologically realistic (Kaehler et al. 2017; Morrison 2006). Computational

1019 limitation is a major factor for implementing directional evolution-models for large datasets that

1020 employ multi-state characters including MSA datasets. Regardless, exclusive reliance on a single

1021 data type, and a single evolutionary process (i.e. substitution mutation) might not be sufficient

1022 for resolving all phylogenetic relationships. Historical signals in MSAs and other data types

1023 relate to qualitatively different, and mutually exclusive evolutionary processes that cannot be

1024 modeled simultaneously. Therefore, polyphasic analyses, rather than a combined analysis of

1025 different data types that are informative at different phylogenetic depths could be useful.

1026

1027 Acknowledgements

1028 I am grateful, foremost, to David Morrison and Charles Kurland for stimulating discussions.

1029 David Morrison, Charles Kurland, Måns Ehrenberg and Suparna Sanyal for support and

1030 encouragement. Siv Andersson for inspiring the article title (in part) and general discussion.

1031 Seraina Klopfstein for providing the algorithms for implementing the directional model in

1032 MrBayes, and for helpful suggestions. David Morrison, David Pollock, David Polly and Kenneth

1033 Halanych for comments on an earlier version of the manuscript as well as Bruce Lieberman, and

1034 Joseph Gillespie for thoughtful comments that helped improve the presentation; two anonymous

1035 reviewers for critique. Thijs Ettema for providing the SSU rRNA and Core-genes-II MSAs, and

1036 Erling Wikman for help with computing equipment.

1037

1038

1039

1040

\section{REFERENCES}

1041

1042

1043

1044

1045

1046

Anantharaman K, Brown CT, Hug LA, Sharon I, Castelle CJ, Probst AJ, Thomas BC, Singh A, Wilkins MJ, Karaoz U, Brodie EL, Williams KH, Hubbard SS, and Banfield JF. 2016. Thousands of microbial genomes shed light on interconnected biogeochemical processes in an aquifer system. Nature Communications 7:13219.

Andreeva A, Howorth D, Chothia C, Kulesha E, and Murzin AG. 2014. SCOP2 prototype: a new approach to protein structure mining. Nucleic Acids Research 42:D310-D314.

Arenas M. 2015. Trends in substitution models of molecular evolution. Frontiers in Genetics $6: 319$. 
1049

1050

1051

1052

1053

1054

1055

1056

1057

1058

1059

1060

1061

1062

1063

1064

1065

1066

1067

1068

1069

1070

1071

1072

1073

1074

1075

1076

1077

1078

1079

1080

1081

Atkinson GC. 2015. The evolutionary and functional diversity of classical and lesser-known cytoplasmic and organellar translational GTPases across the tree of life. BMC Genomics $16: 78$.

Avise JC, and Robinson TJ. 2008. Hemiplasy: a new term in the lexicon of phylogenetics. Systematic Biology 57:503-507.

Baldauf SL, Palmer JD, and Doolittle WF. 1996. The root of the universal tree and the origin of eukaryotes based on elongation factor phylogeny. Proceedings of the National Academy of Sciences of the United States of America 93:7749-7754.

Bergsten J, Nilsson AN, and Ronquist F. 2013. Bayesian tests of topology hypotheses with an example from diving beetles. Systematic Biology 62:660-673.

Boussau B, and Daubin V. 2010. Genomes as documents of evolutionary history. Trends in Ecology and Evolution 25:224-232.

Boyer M, Madoui M-A, Gimenez G, La Scola B, and Raoult D. 2010. Phylogenetic and Phyletic Studies of Informational Genes in Genomes Highlight Existence of a 4th Domain of Life Including Giant Viruses. PloS one 5:e15530.

Brinkmann H, and Philippe H. 1999. Archaea sister group of Bacteria? Indications from tree reconstruction artifacts in ancient phylogenies. Molecular biology and evolution 16:817825.

Castoe TA, de Koning AJ, and Pollock DD. 2010. Adaptive molecular convergence: Molecular evolution versus molecular phylogenetics. Communicative and Integrative Biology 3:1217.

Chothia C, Gough J, Vogel C, and Teichmann SA. 2003. Evolution of the protein repertoire. Science 300:1701-1703.

Coenye T, Gevers D, de Peer YV, Vandamme P, and Swings J. 2005. Towards a prokaryotic genomic taxonomy*. FEMS Microbiology Reviews 29:147-167.

Criscuolo A, and Gribaldo S. 2010. BMGE (Block Mapping and Gathering with Entropy): a new software for selection of phylogenetic informative regions from multiple sequence alignments. BMC Evolutionary Biology 10:210.

Da Cunha V, Gaia M, Gadelle D, Nasir A, and Forterre P. 2017. Lokiarchaea are close relatives of Euryarchaeota, not bridging the gap between prokaryotes and eukaryotes. PLoS genetics 13:e1006810.

Darwin C. 1859. On the Origin of Species by Means of Natural Selection, or the Preservation of Favoured Races in the Struggle for Life. London: John Murray. 
1082 Derelle R, Torruella G, Klimeš V, Brinkmann H, Kim E, Vlček Č, Lang BF, and Eliáš M. 2015.

1083

1084

1085

1086

1087

1088

1089

1090

1091

1092

1093

1094

1095

1096

1097

1098

1099

1100

1101

1102

1103

1104

1105

1106

1107

1108

1109

1110

1111

1112

1113

1114

Bacterial proteins pinpoint a single eukaryotic root. Proceedings of the National Academy of Sciences 112:E693-E699.

Dickinson W. 1995. Molecules and morphology: where's the homology? Trends in Genetics 11:119-121.

Eddy SR. 1998. Profile hidden Markov models. Bioinformatics 14:755-763.

Eisen JA. 1998. Phylogenomics: improving functional predictions for uncharacterized genes by evolutionary analysis. Genome Research 8:163-167.

Eisen JA. 2000. Horizontal gene transfer among microbial genomes: new insights from complete genome analysis. Current opinion in genetics \& development 10:606-611.

Fang H, Oates ME, Pethica RB, Greenwood JM, Sardar AJ, Rackham OJL, Donoghue PCJ, Stamatakis A, De Lima Morais DA, and Gough J. 2013. A daily-updated tree of (sequenced) life as a reference for genome research. Scientific Reports 3.

Finn RD, Attwood TK, Babbitt PC, Bateman A, Bork P, Bridge AJ, Chang H-Y, Dosztányi Z, El-Gebali S, and Fraser M. 2016. InterPro in 2017-beyond protein family and domain annotations. Nucleic Acids Research 45:D190-D199.

Foote AD, Liu Y, Thomas GWC, Vinar T, Alfoldi J, Deng J, Dugan S, van Elk CE, Hunter ME, Joshi V, Khan Z, Kovar C, Lee SL, Lindblad-Toh K, Mancia A, Nielsen R, Qin X, Qu J, Raney BJ, Vijay N, Wolf JBW, Hahn MW, Muzny DM, Worley KC, Gilbert MTP, and Gibbs RA. 2015. Convergent evolution of the genomes of marine mammals. Nat Genet advance online publication.

Forterre P. 1992. Neutral terms. Nature 355:305.

Forterre P, and Philippe H. 1999. Where is the root of the universal tree of life? BioEssays 21:871-879.

Foster PG, Cox CJ, and Embley TM. 2009. The primary divisions of life: a phylogenomic approach employing composition-heterogeneous methods. Philosophical Transactions of the Royal Society B: Biological Sciences 364:2197-2207.

Fuerst JA. 2013. The PVC superphylum: exceptions to the bacterial definition? Antonie Van Leeuwenhoek 104:451-466.

Garrett RA. 1985. Molecular evolution: The uniqueness of Archaebacteria. Nature 318:233-235.

Gough J, Karplus K, Hughey R, and Chothia C. 2001. Assignment of homology to genome sequences using a library of hidden Markov models that represent all proteins of known structure. Journal of Molecular Biology 313:903-919. 
1115 Gouy R, Baurain D, and Philippe H. 2015. Rooting the tree of life: the phylogenetic jury is still $1116 \quad$ out. Phil Trans R Soc B 370:20140329.

1117 Govindarajan S, Recabarren R, and Goldstein RA. 1999. Estimating the total number of protein 1118 folds. Proteins: Structure, Function and Genetics 35:408-414.

1119 Graham DE, Overbeek R, Olsen GJ, and Woese CR. 2000. An archaeal genomic signature. Proceedings of the National Academy of Sciences 97:3304-3308.

1121

1122

1123

1124

1125

1126

1127

1128

1129

1130

1131

1132

1133

1134

1135

1136

1137

1138

1139

1140

1141

1142

1143

1144

1145

1146

1147

Graham SW, Olmstead RG, and Barrett SCH. 2002. Rooting Phylogenetic Trees with Distant Outgroups: A Case Study from the Commelinoid Monocots. Molecular biology and evolution 19:1769-1781.

Gribaldo S, and Brochier-Armanet C. 2012. Time for order in microbial systematics. Trends in microbiology 20:209-210.

Gribaldo S, Poole AM, Daubin V, Forterre P, and Brochier-Armanet C. 2010. The origin of eukaryotes and their relationship with the Archaea: are we at a phylogenomic impasse? Nat Rev Micro 8:743-752.

Guindon S, Dufayard J-F, Lefort V, Anisimova M, Hordijk W, and Gascuel O. 2010. New algorithms and methods to estimate maximum-likelihood phylogenies: assessing the performance of PhyML 3.0. Systematic Biology 59:307-321.

Gupta RS. 2016. Impact of genomics on the understanding of microbial evolution and classification: the importance of Darwin's views on classification. FEMS Microbiology Reviews 40:520-553.

Guy L, and Ettema TJG. 2011. The archaeal TACK superphylum and the origin of eukaryotes. Trends in microbiology 19:580-587.

Harish A, and Kurland CG. 2017a. Akaryotes and Eukaryotes are independent descendants of a universal common ancestor. Biochimie 138:168-183.

Harish A, and Kurland CG. 2017b. Empirical genome evolution models root the tree of life. Biochimie 138:137-155.

Harish A, and Kurland CG. 2017c. Mitochondria are not captive bacteria. Journal of theoretical biology 434:88-98.

Harish A, Tunlid A, and Kurland CG. 2013. Rooted phylogeny of the three superkingdoms. Biochimie 95:1593-1604.

He D, Fiz-Palacios O, Fu CJ, Tsai CC, and Baldauf SL. 2014. An alternative root for the eukaryote tree of life. Current Biology 24:465-470.

Hennig W. 1965. Phylogenetic systematics. Annual review of entomology 10:97-116.

PeerJ reviewing PDF | (2018:06:28733:2:0:NEW 27 Aug 2018) 
1148 Hillis DM. 1999. SINEs of the perfect character. Proceedings of the National Academy of $1149 \quad$ Sciences 96:9979-9981.

1150 Huelsenbeck JP, Bollback JP, and Levine AM. 2002. Inferring the root of a phylogenetic tree. $1151 \quad$ Systematic Biology 51:32-43.

1152 Huelsenbeck JP, Larget B, and Alfaro ME. 2004. Bayesian Phylogenetic Model Selection Using Reversible Jump Markov Chain Monte Carlo. Molecular biology and evolution 21:11231133.

1155

1156

Hug LA, Baker BJ, Anantharaman K, Brown CT, Probst AJ, Castelle CJ, Butterfield CN, Hernsdorf AW, Amano Y, and Ise K. 2016. A new view of the tree of life. Nature Microbiology 1:16048.

Huson DH, and Bryant D. 2006. Application of phylogenetic networks in evolutionary studies. Mol Biol Evol 23.

Iwabe N, Kuma K, Hasegawa M, Osawa S, and Miyata T. 1989. Evolutionary relationship of archaebacteria, eubacteria, and eukaryotes inferred from phylogenetic trees of duplicated genes. Proceedings of the National Academy of Sciences 86:9355-9359.

Kaehler BD, Yap VB, and Huttley GA. 2017. Standard codon substitution models overestimate purifying selection for nonstationary data. Genome Biology and Evolution 9:134-149.

Kalyaanamoorthy S, Minh BQ, Wong TK, von Haeseler A, and Jermiin LS. 2017. ModelFinder: fast model selection for accurate phylogenetic estimates. Nature methods 14:587.

Kass RE, and Raftery AE. 1995. Bayes factors. Journal of the american statistical association 90:773-795.

Kim KM, and Caetano-Anollés G. 2011. The proteomic complexity and rise of the primordial ancestor of diversified life. BMC Evolutionary Biology 11.

Klopfstein S, Vilhelmsen L, and Ronquist F. 2015. A Nonstationary Markov Model Detects Directional Evolution in Hymenopteran Morphology. Systematic Biology 64:1089-1103.

Kurland CG, and Andersson SGE. 2000. Origin and evolution of the mitochondrial proteome. Microbiology and Molecular Biology Reviews 64:786-820.

Kurland CG, Canback B, and Berg OG. 2003. Horizontal gene transfer: A critical view. Proceedings of the National Academy of Sciences of the United States of America 100:9658-9662.

1179

Lake JA. 1986. An alternative to archaebacterial dogma. Nature 319:626-626.

Lake JA, Henderson E, Oakes M, and Clark MW. 1984. Eocytes: a new ribosome structure indicates a kingdom with a close relationship to eukaryotes. Proceedings of the National Academy of Sciences 81:3786-3790. 
1182 Lake JA, Jain R, and Rivera MC. 1999. Mix and Match in the Tree of Life. Science 283:202711832028.

1184 Lartillot N, and Philippe H. 2004. A Bayesian mixture model for across-site heterogeneities in 1185 the amino-acid replacement process. Mol Biol Evol 21:1095-1109.

1186 Le SQ, and Gascuel O. 2008. An Improved General Amino Acid Replacement Matrix.

$1187 \quad$ Molecular biology and evolution 25:1307-1320.

1188 Lefort V, Longueville J-E, and Gascuel O. 2017. SMS: Smart Model Selection in PhyML.

$1189 \quad$ Molecular biology and evolution:msx149.

1190 Lewis PO. 2001. A Likelihood Approach to Estimating Phylogeny from Discrete Morphological

$1191 \quad$ Character Data. Systematic Biology 50:913-925.

1192 Light S, Sagit R, Sachenkova O, Ekman D, and Elofsson A. 2013. Protein expansion is primarily due to indels in intrinsically disordered regions. Molecular biology and evolution 30:2645-2653.

1195

1196

1197

1198

1199

Liu Y, Cotton JA, Shen B, Han X, Rossiter SJ, and Zhang S. 2010. Convergent sequence evolution between echolocating bats and dolphins. Current Biology 20:R53-R54.

Mackin KA, Roy RA, and Theobald DL. 2014. An empirical test of convergent evolution in rhodopsins. Molecular biology and evolution 31:85-95.

Martin W. 1999. Mosaic bacterial chromosomes: a challenge en route to a tree of genomes. BioEssays 21:99-104.

1201

1202

1203

1204

1205

1206

Mayr E. 1998. Two empires or three? Proceedings of the National Academy of Sciences of the United States of America 95:9720-9723.

Mistry J, Finn RD, Eddy SR, Bateman A, and Punta M. 2013. Challenges in homology search: HMMER3 and convergent evolution of coiled-coil regions. Nucleic Acids Research 41:e121-e121.

Morrison DA. 2006. Phylogenetic Analyses of Parasites in the New Millennium. Advances in Parasitology. p 1-124.

Morrison DA. 2009. Using data-display networks for exploratory data analysis in phylogenetic studies. Molecular biology and evolution 27:1044-1057.

Morrison DA, Morgan M, and Kelchner M. 2015. Molecular homology and multiple sequence alignment: an analysis of concepts and practice. Australian Systematic Botany In press.

1212 Murray GGR, Weinert LA, Rhule EL, and Welch JJ. 2016. The Phylogeny of Rickettsia Using Different Evolutionary Signatures: How Tree-Like is Bacterial Evolution? Systematic Biology 65:265-279. 
1215 Murzin AG, Brenner SE, Hubbard T, and Chothia C. 1995. SCOP: A structural classification of

1216

1217

1218

1219

1220

1221

1222

1223

1224

1225

1226

1227

1228

1229

1230

1231

1232

1233

1234

1235

1236

1237

1238

1239

1240

1241

1242

1243

1244

1245

1246

1247

1248

1249 proteins database for the investigation of sequences and structures. Journal of Molecular Biology 247:536-540.

Nasir A, and Caetano-Anollés G. 2015. A phylogenomic data-driven exploration of viral origins and evolution. Science Advances 1.

Nelson-Sathi S, Sousa FL, Roettger M, Lozada-Chavez N, Thiergart T, Janssen A, Bryant D, Landan G, Schonheit P, Siebers B, McInerney JO, and Martin WF. 2015. Origins of major archaeal clades correspond to gene acquisitions from bacteria. Nature 517:77-80.

Oates ME, Stahlhacke J, Vavoulis DV, Smithers B, Rackham OJL, Sardar AJ, Zaucha J, Thurlby N, Fang H, and Gough J. 2015. The SUPERFAMILY 1.75 database in 2014: A doubling of data. Nucleic Acids Research 43:D227-D233.

Padian K, Lindberg DR, and Polly PD. 1994. Cladistics and the fossil record: the uses of history. Annual Review of Earth and Planetary Sciences 22:63-91.

Park J, Karplus K, Barrett C, Hughey R, Haussler D, Hubbard T, and Chothia C. 1998. Sequence comparisons using multiple sequences detect three times as many remote homologues as pairwise methods. Journal of Molecular Biology 284:1201-1210.

Parker J, Tsagkogeorga G, Cotton JA, Liu Y, Provero P, Stupka E, and Rossiter SJ. 2013. Genome-wide signatures of convergent evolution in echolocating mammals. Nature 502:228-231.

Parks DH, Rinke C, Chuvochina M, Chaumeil P-A, Woodcroft BJ, Evans PN, Hugenholtz P, and Tyson GW. 2017. Recovery of nearly 8,000 metagenome-assembled genomes substantially expands the tree of life. Nature Microbiology.

Pearson WR, and Sierk ML. 2005. The limits of protein sequence comparison? Current Opinion in Structural Biology 15:254-260.

Pechman KJ, and Woese CR. 1972. Characterization of the primary structural homology between the $16 \mathrm{~S}$ ribosomal RNAs ofEscherichia coli andBacillus megaterium by oligomer cataloging. Journal of Molecular Evolution 1:230-240.

Pethica RB, Levitt M, and Gough J. 2012. Evolutionarily consistent families in SCOP: Sequence, structure and function. BMC Structural Biology 12.

Philippe H, Brinkmann H, Lavrov DV, Littlewood DTJ, Manuel M, Wörheide G, and Baurain D. 2011. Resolving difficult phylogenetic questions: Why more sequences are not enough. PLoS Biology 9.

Rinke C, Schwientek P, Sczyrba A, Ivanova NN, Anderson IJ, Cheng J-F, Darling A, Malfatti S, Swan BK, Gies EA, Dodsworth JA, Hedlund BP, Tsiamis G, Sievert SM, Liu W-T, Eisen JA, Hallam SJ, Kyrpides NC, Stepanauskas R, Rubin EM, Hugenholtz P, and Woyke T.

Peer] reviewing PDF | (2018:06:28733:2:0:NEW 27 Aug 2018) 
1250

1251

1252

1253

1254

1255

1256

1257

1258

1259

1260

1261

1262

1263

1264

1265

1266

1267

1268

1269

1270

1271

1272

1273

1274

1275

1276

1277

1278

1279

1280

1281
2013. Insights into the phylogeny and coding potential of microbial dark matter. Nature 499:431-437.

Rochette NC, Brochier-Armanet C, and Gouy M. 2014. Phylogenomic test of the hypotheses for the evolutionary origin of eukaryotes. Molecular biology and evolution 31:832-845.

Roettger M, Martin W, and Dagan T. 2009. A Machine-Learning Approach Reveals That Alignment Properties Alone Can Accurately Predict Inference of Lateral Gene Transfer from Discordant Phylogenies. Molecular biology and evolution 26:1931-1939.

Rokas A, and Carroll SB. 2006. Bushes in the tree of life. PLoS Biology 4:1899-1904.

Rokas A, and Carroll SB. 2008. Frequent and widespread parallel evolution of protein sequences. Molecular biology and evolution 25:1943-1953.

Rokas A, and Holland PWH. 2000. Rare genomic changes as a tool for phylogenetics. Trends in Ecology and Evolution 15:454-459.

Ronquist F, Huelsenbeck J, and Teslenko M. 2011. MrBayes version 3.2 manual: tutorials and model summaries. Available with the software distribution at mrbayessourceforgenet/mb32_manualpdf.

Ronquist F, Teslenko M, van der Mark P, Ayres DL, Darling A, Höhna S, Larget B, Liu L, Suchard MA, and Huelsenbeck JP. 2012. MrBayes 3.2: Efficient Bayesian Phylogenetic Inference and Model Choice Across a Large Model Space. Systematic Biology 61:539542.

Sagan L. 1967. On the origin of mitosing cells. Journal of theoretical biology 14:225-275.

Salichos L, and Rokas A. 2013. Inferring ancient divergences requires genes with strong phylogenetic signals. Nature 497:327-331.

Salzberg SL. 2017. Horizontal gene transfer is not a hallmark of the human genome. Genome Biology 18:85.

Salzberg SL, White O, Peterson J, and Eisen JA. 2001. Microbial genes in the human genome: Lateral transfer or gene loss? Science 292:1903-1906.

Sanderson MJ. 2005. Where have all the clades gone? A systematist's take in Inferring Phylogenies. Evolution 59:2056-2058.

Schwartz R, and Dayhoff M. 1978. Origins of prokaryotes, eukaryotes, mitochondria, and chloroplasts. Science 199:395-403.

Shen X-X, Hittinger CT, and Rokas A. 2017. Contentious relationships in phylogenomic studies can be driven by a handful of genes. Nature ecology \& evolution 1:0126. 
1282 Snel B, Bork P, and Huynen MA. 1999. Genome phylogeny based on gene content. Nature $1283 \quad$ Genetics 21:108-110.

1284

1285

1286

1287

1288

1289

1290

1291

1292

1293

1294

1295

1296

1297

1298

1299

1300

1301

1302

1303

1304

1305

1306

1307

1308

1309

1310

1311

1312

1313

1314

1315

1316

Spang A, Saw JH, Jørgensen SL, Zaremba-Niedzwiedzka K, Martijn J, Lind AE, van Eijk R, Schleper C, Guy L, and Ettema TJ. 2015. Complex archaea that bridge the gap between prokaryotes and eukaryotes. Nature 521:173-179.

Stanier RY, and Niel Cv. 1962. The concept of a bacterium. Archives of Microbiology 42:17-35.

Sunagawa S, Coelho LP, Chaffron S, Kultima JR, Labadie K, Salazar G, Djahanschiri B, Zeller G, Mende DR, and Alberti A. 2015. Structure and function of the global ocean microbiome. Science 348:1261359.

Susko E, and Roger AJ. 2007. On reduced amino acid alphabets for phylogenetic inference. Molecular biology and evolution 24:2139-2150.

Tarver JE, Sperling EA, Nailor A, Heimberg AM, Robinson JM, King BL, Pisani D, Donoghue PC, and Peterson KJ. 2013. miRNAs: small genes with big potential in metazoan phylogenetics. Molecular biology and evolution 30:2369-2382.

Tatusov RL, Galperin MY, Natale DA, and Koonin EV. 2000. The COG database: a tool for genome-scale analysis of protein functions and evolution. Nucleic Acids Research 28:3336 .

Thiergart T, Landan G, Schenk M, Dagan T, and Martin WF. 2012. An evolutionary network of genes present in the eukaryote common ancestor polls genomes on eukaryotic and mitochondrial origin. Genome Biology and Evolution 4:466-485.

Tourasse NJ, and Gouy M. 1999. Accounting for evolutionary rate variation among sequence sites consistently changes universal phylogenies deduced from rRNA and protein-coding genes. Molecular Phylogenetics and Evolution 13:159-168.

Valentine DL. 2007. Adaptations to energy stress dictate the ecology and evolution of the Archaea. Nature Reviews Microbiology 5:316-323.

Wang M, and Caetano-Anolles G. 2006. Global phylogeny determined by the combination of protein domains in proteomes. Mol Biol Evol 23:2444-2454.

Wang M, Kurland CG, and Caetano-Anollés G. 2011. Reductive evolution of proteomes and protein structures. Proceedings of the National Academy of Sciences of the United States of America 108:11954-11958.

Wheeler Q, Assis L, and Rieppel O. 2013. Phylogenetics: Heed the father of cladistics. Nature 496:295-296.

Whelan NV, Kocot KM, Moroz LL, and Halanych KM. 2015. Error, signal, and the placement of Ctenophora sister to all other animals. Proceedings of the National Academy of Sciences 112:5773-5778. 
1317 Whittaker RH. 1969. New concepts of kingdoms of organisms. Science 163:150-160.

1318 Wiley EO, and Lieberman BS. 2011. Phylogenetics: theory and practice of phylogenetic 1319 systematics: John Wiley \& Sons.

1320 Williams TA, and Embley TM. 2014. Archaeal "dark matter" and the origin of eukaryotes. $1321 \quad$ Genome Biology and Evolution 6:474-481.

1322 Williams TA, Foster PG, Cox CJ, and Embley TM. 2013. An archaeal origin of eukaryotes supports only two primary domains of life. Nature 504:231-236.

1324 Woese CR. 1987. Bacterial evolution. Microbiological reviews 51:221.

1325

1326

1327

1328

1329

1330

1331

1332

1333

1334

1335

1336

1337

1338

1339

1340

1341

1342

1343

1344

1345

1346

1347

1348

1349

1350
Woese CR. 2002. On the evolution of cells. Proceedings of the National Academy of Sciences of the United States of America 99:8742-8747.

Woese CR. 2004. The Archaeal Concept and the World it Lives in: A Retrospective. Photosynthesis Research 80:361-372.

Woese CR, and Fox GE. 1977. Phylogenetic structure of the prokaryotic domain: the primary kingdoms. Proceedings of the National Academy of Sciences 74:5088-5090.

Woese CR, Fox GE, Zablen L, Uchida T, Bonen L, Pechman K, Lewis BJ, and Stahl D. 1975. Conservation of primary structure in $16 \mathrm{~S}$ ribosomal RNA. Nature 254:83-86.

Woese CR, Kandler O, and Wheelis ML. 1990. Towards a natural system of organisms: Proposal for the domains Archaea, Bacteria, and Eucarya. Proceedings of the National Academy of Sciences of the United States of America 87:4576-4579.

Wright AM, and Hillis DM. 2014. Bayesian Analysis Using a Simple Likelihood Model Outperforms Parsimony for Estimation of Phylogeny from Discrete Morphological Data. PloS one 9:e109210.

Wu D, Wu M, Halpern A, Rusch DB, Yooseph S, Frazier M, Venter JC, and Eisen JA. 2011. Stalking the Fourth Domain in Metagenomic Data: Searching for, Discovering, and Interpreting Novel, Deep Branches in Marker Gene Phylogenetic Trees. PloS one 6:e18011.

Yang S, Doolittle RF, and Bourne PE. 2005. Phylogeny determined by protein domain content. Proceedings of the National Academy of Sciences of the United States of America 102:373-378.

Yang Z, and Roberts D. 1995. On the use of nucleic acid sequences to infer early branchings in the tree of life. Molecular biology and evolution 12:451-458.

Zamani-Dahaj SA, Okasha M, Kosakowski J, and Higgs PG. 2016. Estimating the Frequency of Horizontal Gene Transfer Using Phylogenetic Models of Gene Gain and Loss. Molecular biology and evolution:msw062. 
1351 Zaremba-Niedzwiedzka K, Caceres EF, Saw JH, Bäckström D, Juzokaite L, Vancaester E, Seitz 1352 KW, Anantharaman K, Starnawski P, Kjeldsen KU, Stott MB, Nunoura T, Banfield JF, 1353 Schramm A, Baker BJ, Spang A, and Ettema TJG. 2017. Asgard archaea illuminate the 1354 origin of eukaryotic cellular complexity. Nature 541:353-358.

1355 Zuckerkandl E, and Pauling L. 1965. Molecules as documents of evolutionary history. Journal of theoretical biology 8:357-366.

1357 Zwickl DJ, and Hillis DM. 2002. Increased taxon sampling greatly reduces phylogenetic error. $1358 \quad$ Systematic Biology 51:588-598.

1359

1360 


\section{Figure 1 (on next page)}

Data-display networks (DDN) depicting the character conflicts in datasets that employ different character types: nucleotides or amino acids, to resolve the Tree of Life.

(A) SSU rRNA alignment of 1,462 characters. Concatenated amino acid sequence alignment of: (A) 29 genes, 8,563 characters (Core-genes-I dataset); (C) 48 genes, 9,868 characters (Core-genes-II dataset); and (D) SR4 recoded core-genes-II dataset (data simplified from 20 to 4 character-states). Each network is constructed from a neighbor-net analysis based on the observed genetic distance ( $p$-distance) and displayed as an equal angle split network. Edge (branch) lengths correspond to the support for character bipartitions (splits), and reticulations in the tree correspond to character conflicts. The scale bar represents the split support for the edges. Conflicts in character partitions that are incongruent with a tree appear as reticulations in the DDN. Source of the datasets is as specified in Table 1. 
A Peerl Archaea

A PeerJ

B

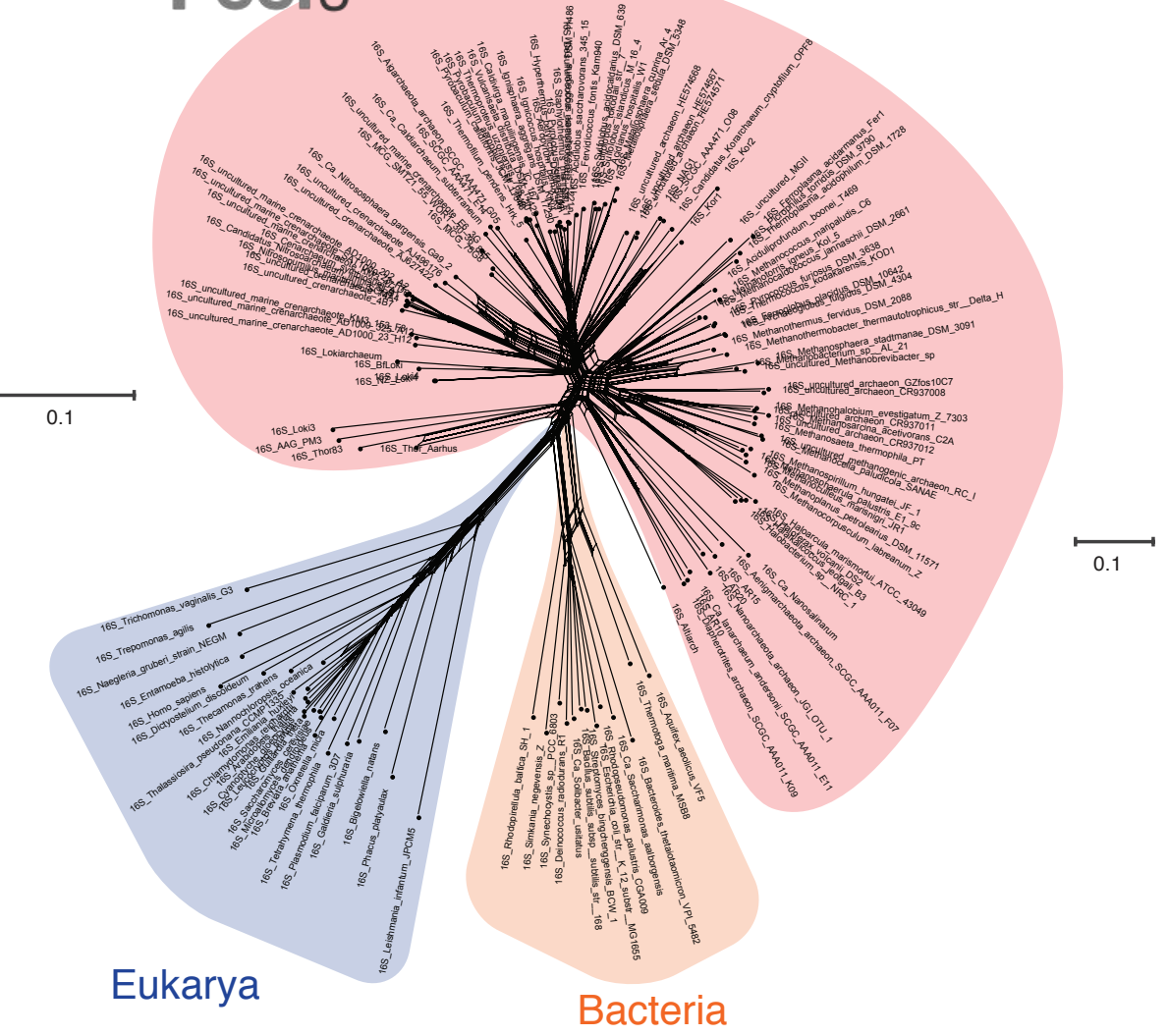

SSU rRNA:

140 taxa; 1,462 characters

C

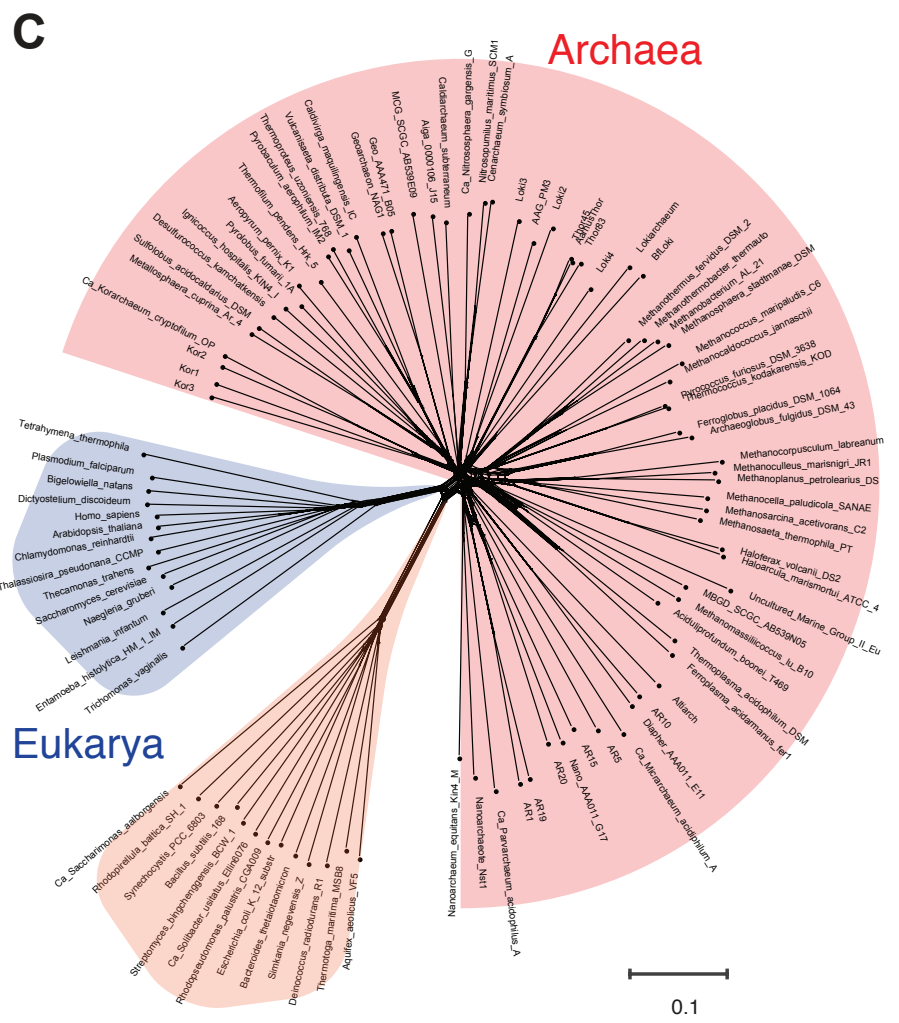

Bacteria

Core-genes-II

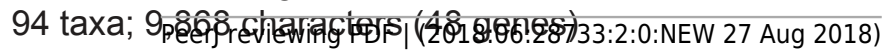

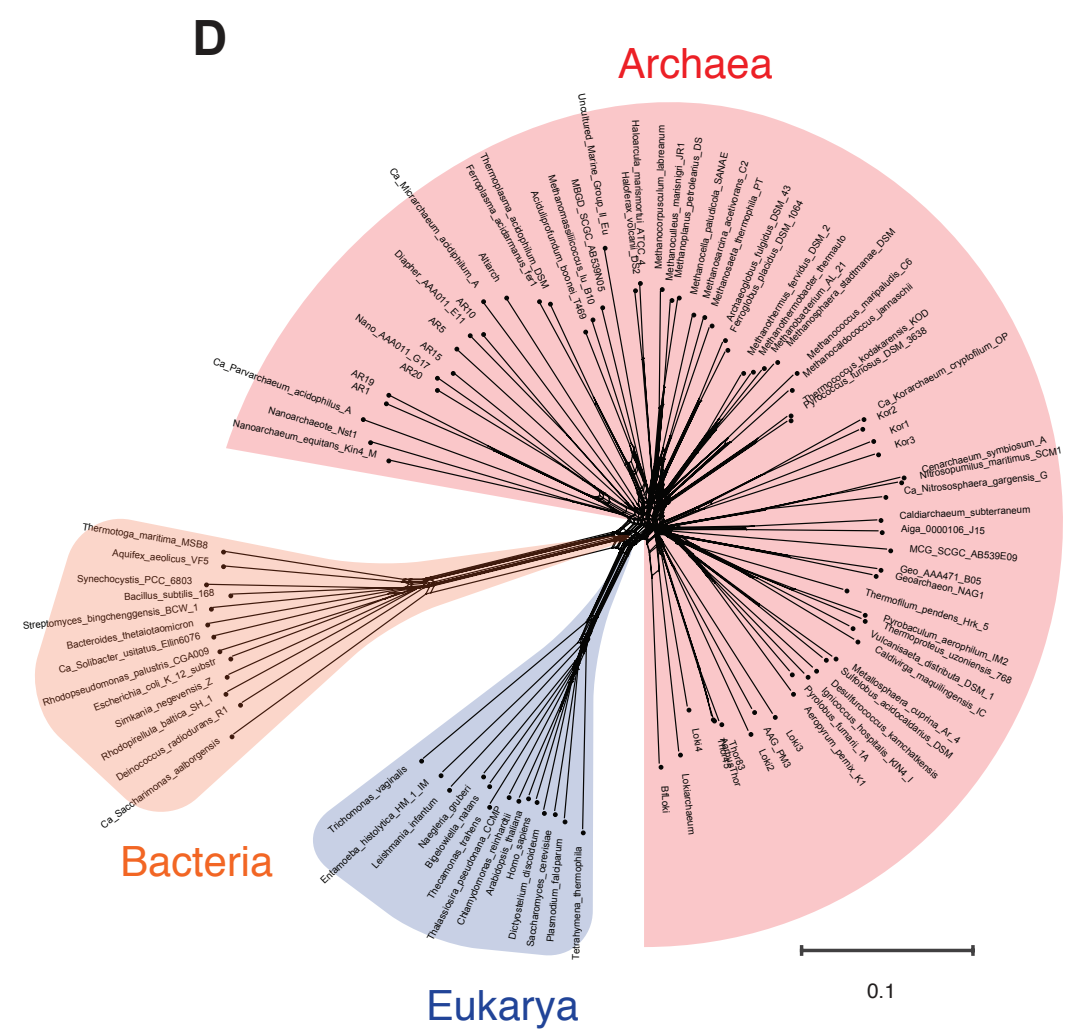

Core-genes-II:

94 taxa; 9,868 characters, SR4 recoded (48 genes) 


\section{Figure 2 (on next page)}

Data-display networks (DDN) depicting character conflicts among complex molecular characters.

Complex characters here are genomic loci that correspond to protein-domains as opposed to elementary characters (individual nucleotides or amino acids). The presence-absence patterns of homologous protein-domains identified by the SCOP (Structural Classification of Proteins) scheme were coded with nonarbitrary state labels to assemble a data matrix. Each network is constructed from a neighbor-net analysis based on the Hamming distance identical to p-distance in (Fig. 1) and displayed as an equal angle split network. (A) DDN of 1,732 characters sampled from 141 species, each from distinct genera (SCOP-I dataset). (B) DDN based on an updated SCOP-I data matrix to include recently described novel species of archaea and bacteria, totaling to 222 species and a modest increase to 1,738 characters (SCOP-II dataset). Details of the DDNs are as in Figure 1. 
A

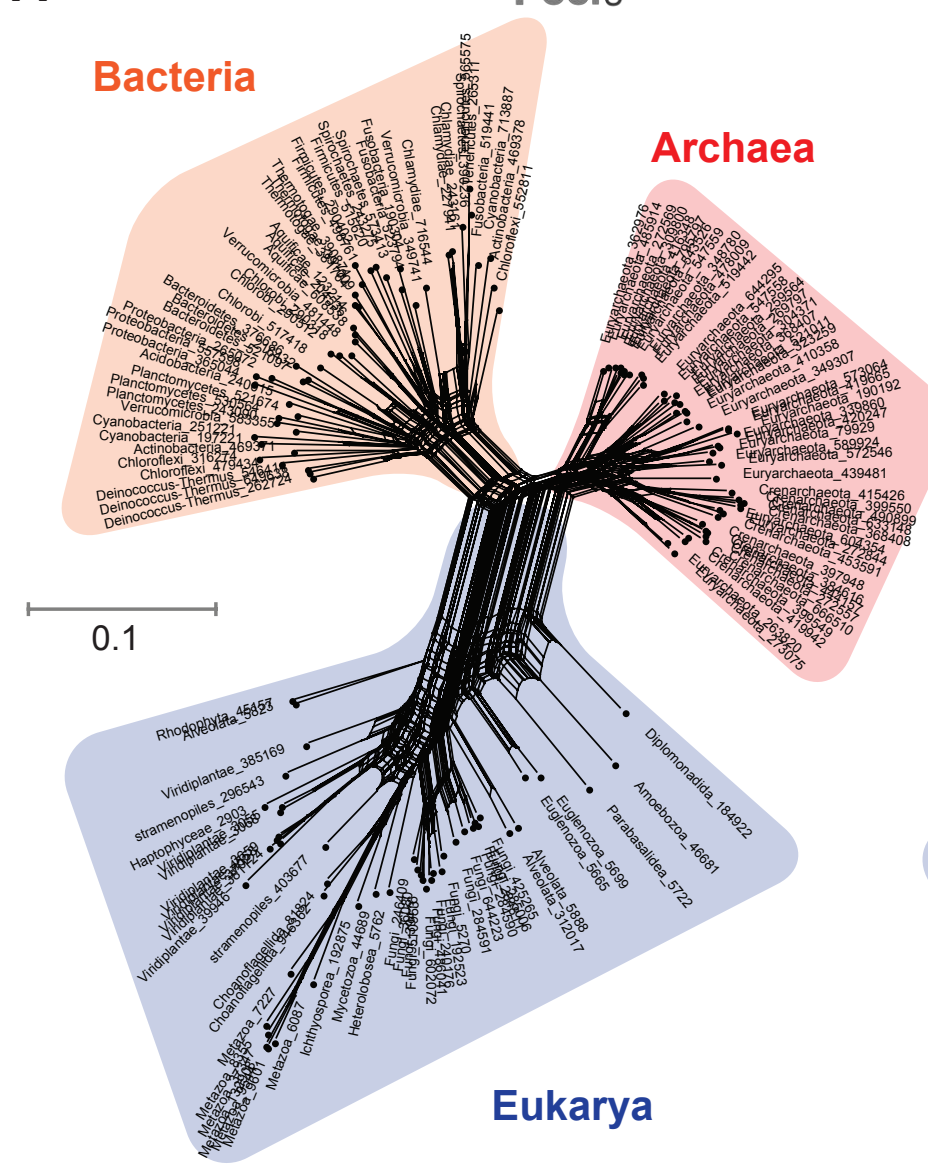

Dataset: SCOP-I

141 taxa; 1,732 characters
B Manuscript to be reviewed

\section{Bacteria}
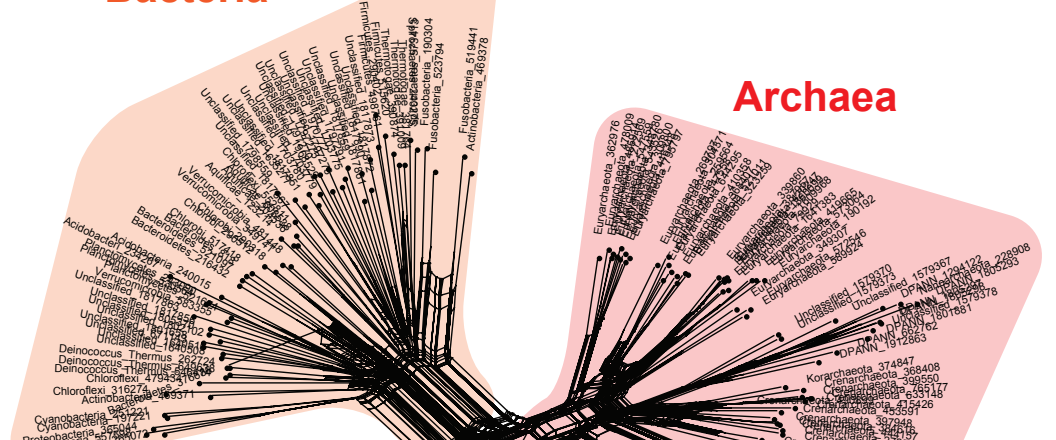

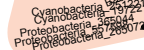

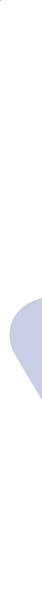

Peer) reviewing PDF | (2018:06:28733:2:0:NEW 27 Aug 2018) 


\section{Figure 3 (on next page)}

Alignment uncertainty in closely related proteins due to domain recombination.

Multi-domain architecture (MDA), the $\mathrm{N}$ - to $\mathrm{C}$-terminal sequence of the translational GTPase superfamily based on recombination of 8 modular domains is shown as (A) linear sequences and (B) 3D structures. 57 distinct families with varying MDAs are known, of which 6 canonical families are shown as a schematic in (A) and the corresponding $3 \mathrm{D}$ folds in (B). Amino acid sequences of only 2 of the 8 conserved domains can be aligned with confidence for use in MSA-based phylogenomics. The length of the alignment varies from 200-300 amino acids depending on the sequence diversity sampled $(13,32)$. The EF-Tu-EF-G paralogous pair employed as pseudo-outgroups for the classical rooting of the rRNA tree is highlighted. (C) Phyletic distribution of 1,738 out the 2,000 distinct SCOP-domains sampled from 222 species used for phylogenetic analyses in the present study. About 70 percent of the domains are widely distributed across the sampled taxonomic diversity. (D) Comparison of the number of genomic loci represented in the different data matrices used in phylogenomic studies. 
A

\section{Peeru}

\section{a/e IF2 $\gamma$}

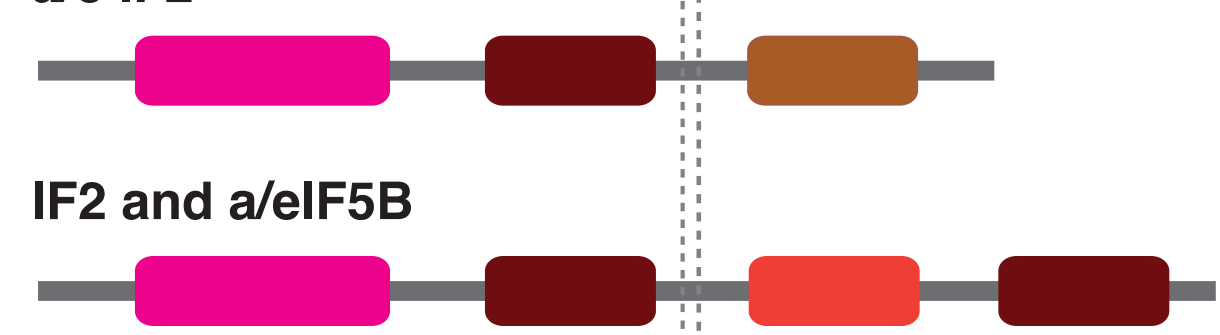

ii. Elongations Factors (EF)

\section{EF-Tu}

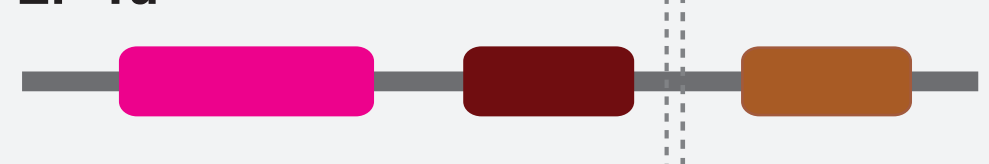

\section{EF-G}

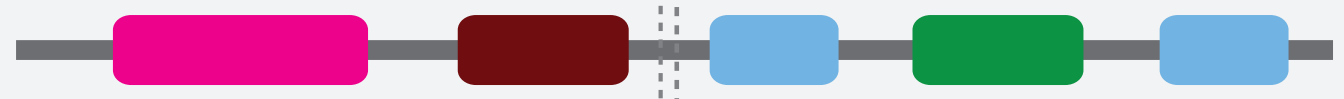

\section{EF-Sec}

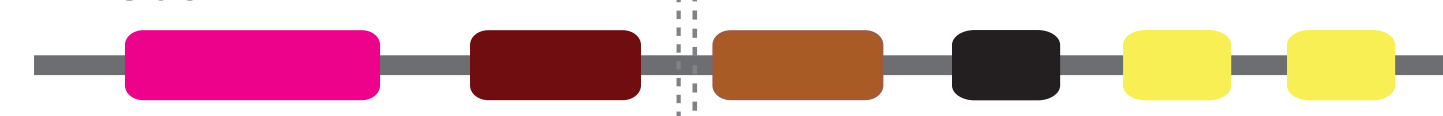

\section{iii. Release Factors (RF)}

\section{RF3}

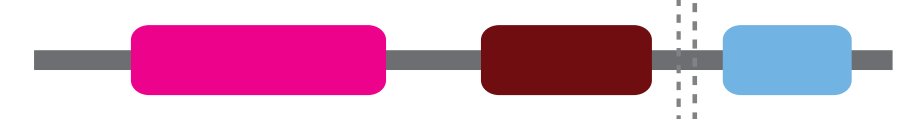

\section{More conserved}

(Reliable MSA)

\section{Less conserved}

(Trimmed out of MSA)

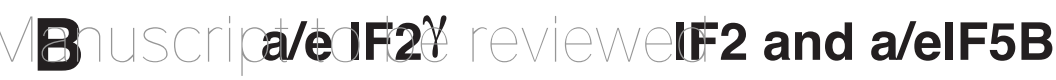
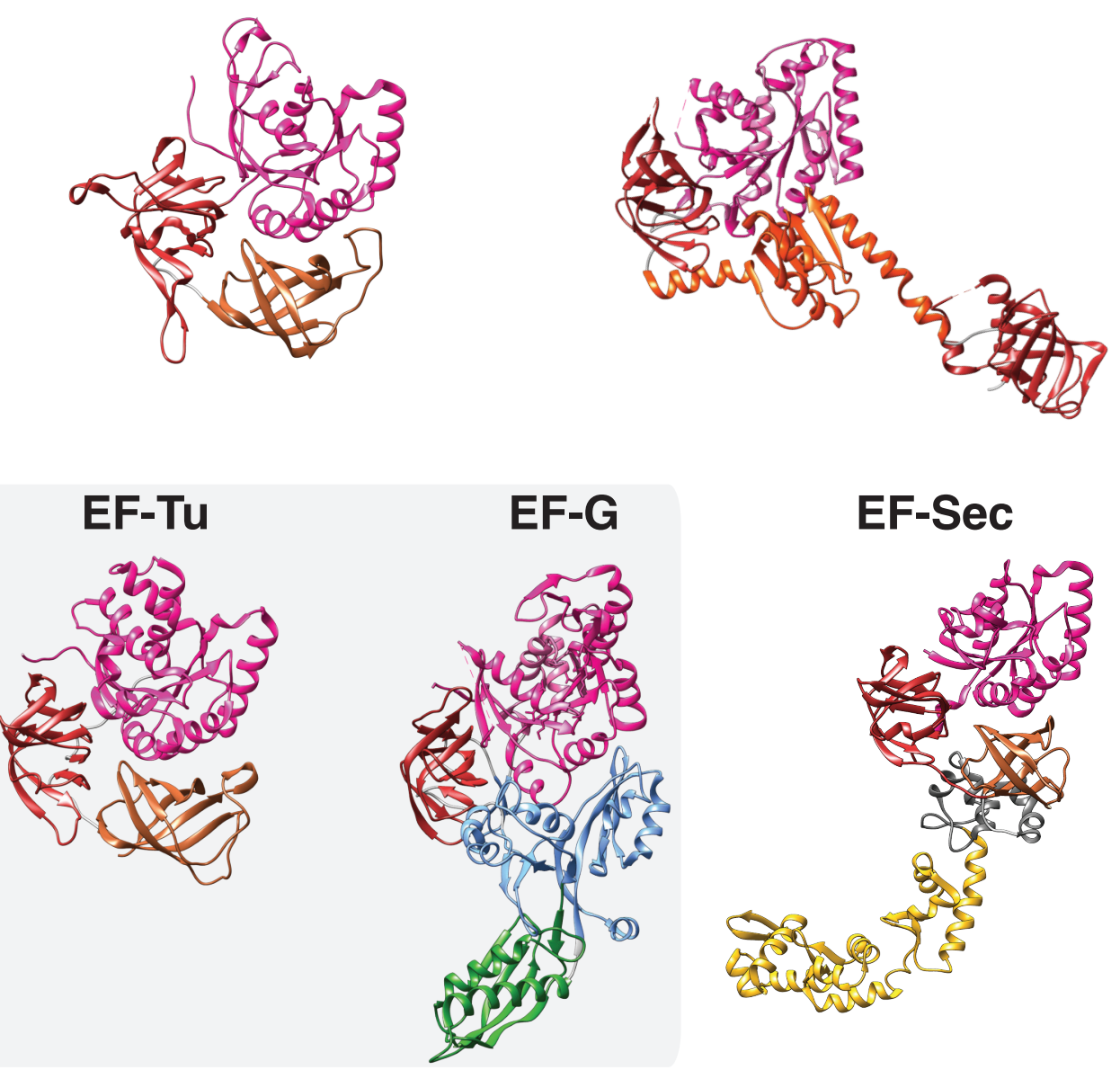

\section{RF3}

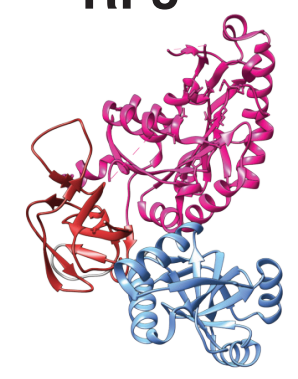

Modular protein-domains classified according to SCOP

P-loop containing NTP hydrolases

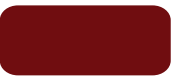

Translation proteins

Initiation factor IF2/elF5b, domain 3

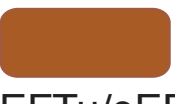

EFTu/eEF1-alpha/elF
Ribosomal protein S5 domain 2like

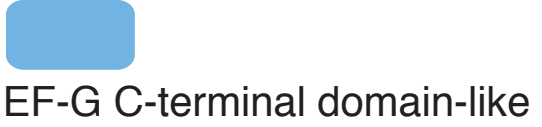

Winged helix DNA-binding domain

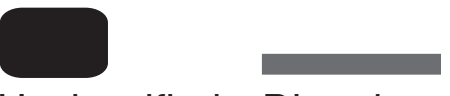

C

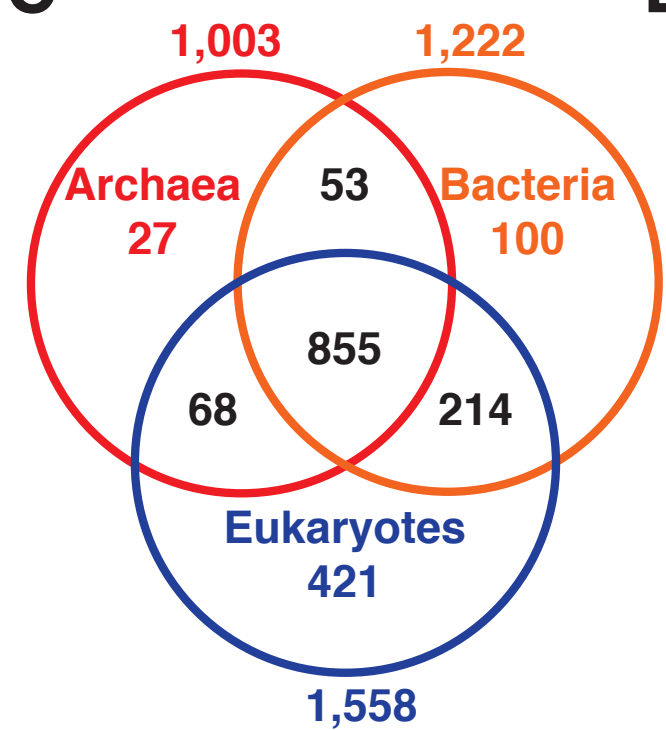

Total: 1,738 domains
D

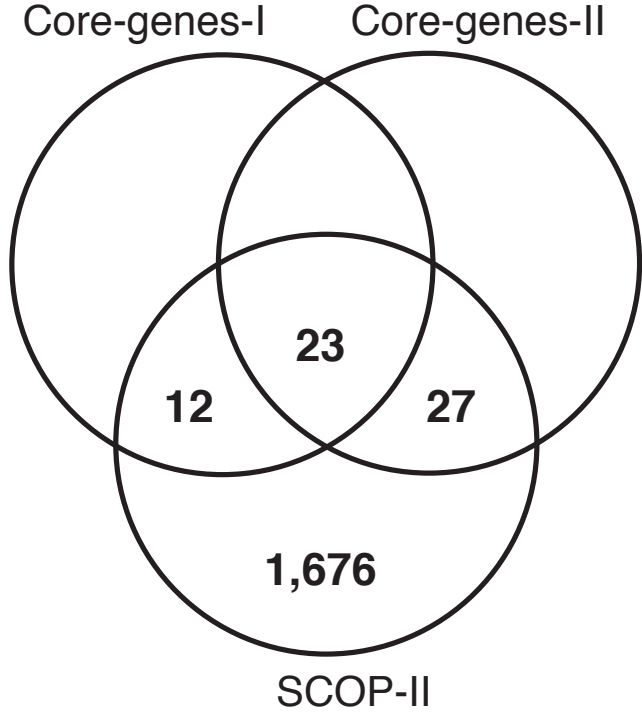

Total: $\mathbf{1 , 7 3 8}$ domains 


\section{Figure 4(on next page)}

Comparison of the sensitivity of the tree topology to character-specific rate heterogeneity (CSRH).

(A-C) Concatenated gene trees derived from amino acid characters, and (D-F) genome trees derived from protein-domain characters. (A, B) Unrooted trees estimated using the coregenes-I dataset for which (A) rate homogeneous-LG model, or (B) a CSRH-LG substitution model was implemented. Branch support values are approximate likelihood-ratio test (aLRT) scores (C) Model-fit to data is ranked according the log likelihood ratio (LLR) scores for the tree topology. LLR scores are computed as the difference from the best-fitting model ( $L G+G 12)$ of the likelihood scores estimated in PhyML. Thus, larger LLR values indicate lesser support for that model/tree, relative to the most-likely model/tree. Substitution rate heterogeneity is approximated with 4, 8 or 12 rate categories in the complex models, but with a single rate category in the simpler model. (C, D) Genome trees derived from the SCOP-II datasets using (C) a rate homogeneous- or, (D) CSRH model of evolution of genomic protein-domain cohorts. Scale bars represent the estimated number of character-state changes. Branch support values are posterior probability (PP) scores estimated in MrBayes. (F) Model fit to data is ranked according log Bayes factor (LBF) scores, which like LLR scores are the log odds of the hypotheses. LBF scores are computed as the difference in likelihood scores estimated in MrBayes. 


\section{A}

A
Eukarya

Peery

$L G$

B

Bacteria
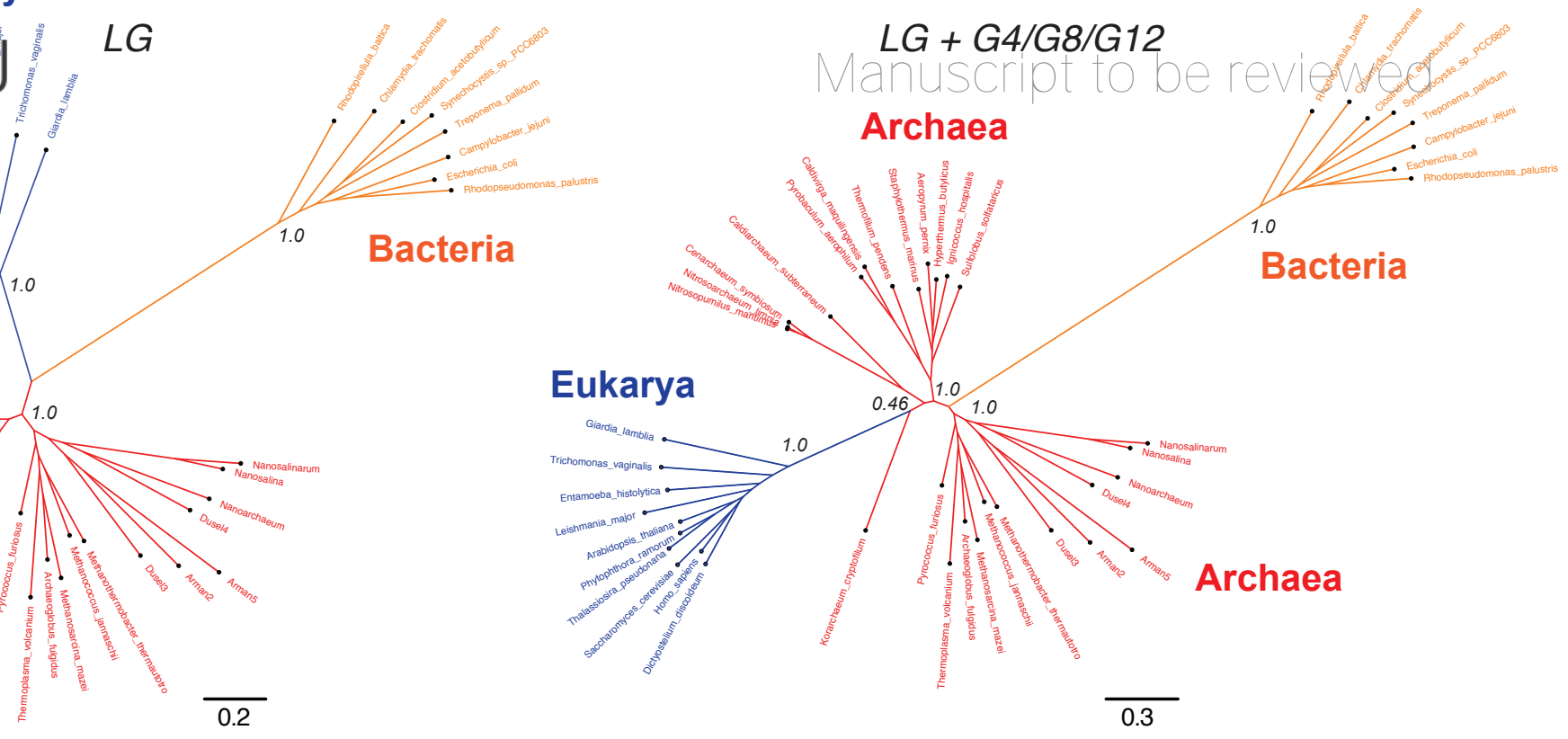

D

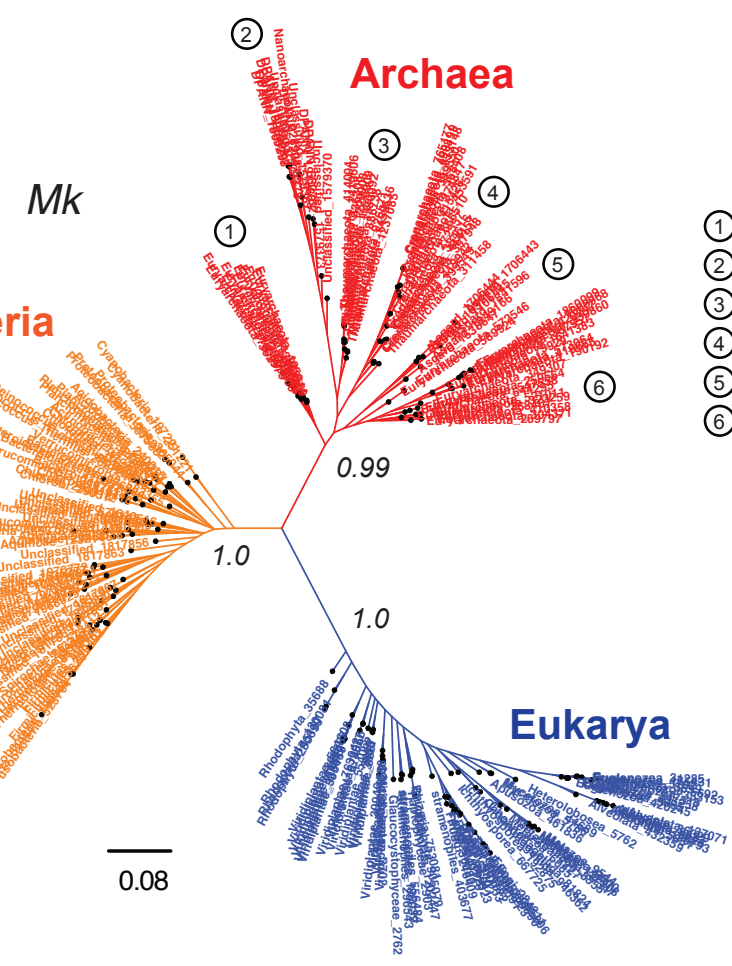

(1) Euryarchaeota

(2) DPANN

(3) Thaumarchaeota

(4) Crenarchaeota

(5) Asgard

(6) Euryarchaeota
E $M k+$ G4/G8/G12

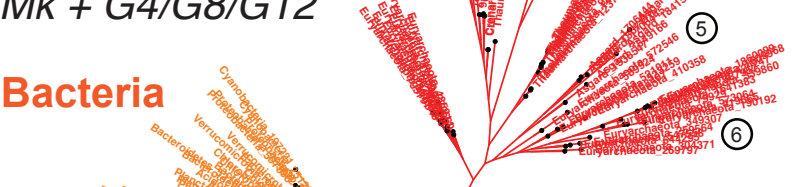

Archaea

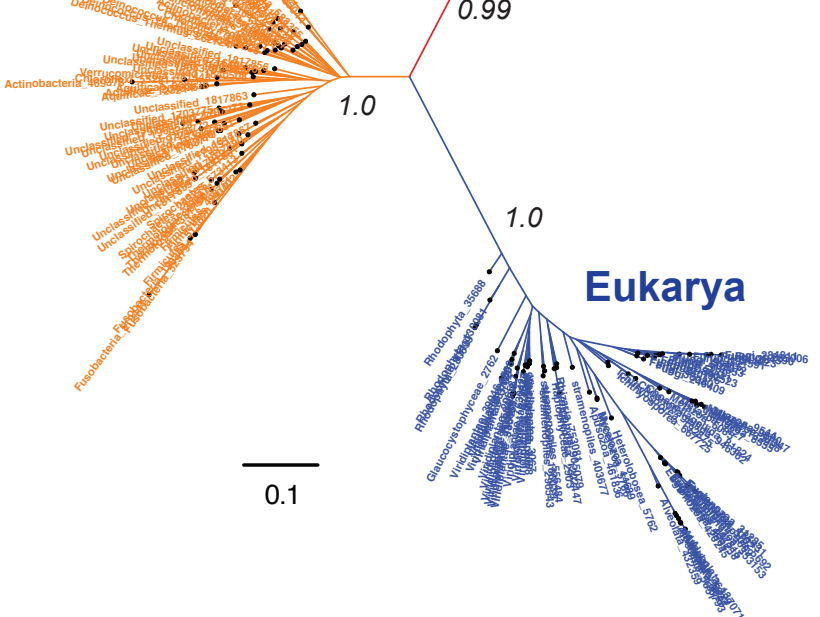

\begin{tabular}{|c|c|c|c|c|c|c|}
\hline $\mathbf{F}$ & No. of taxa & $\begin{array}{l}\text { No. of } \\
\text { characters }\end{array}$ & $\begin{array}{l}\text { Evolution } \\
\text { model }\end{array}$ & $\begin{array}{l}\text { Archaeal } \\
\text { radiation }\end{array}$ & $\begin{array}{l}\text { Likelihood } \\
\text { score }\end{array}$ & LBF \\
\hline & & & $\mathrm{Mk}+\mathrm{G} 12$ & Monophyletic & -79716 & 0 \\
\hline & & & $\mathrm{Mk}+\mathrm{G} 8$ & Monophyletic & -79735 & 19 \\
\hline & 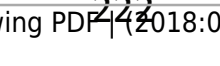 & & 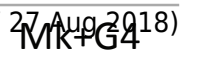 & Monophyletic & -79858 & 142 \\
\hline & & & Mk & Monophyletic & -85817 & 6101 \\
\hline
\end{tabular}




\section{Figure $\mathbf{5}$ (on next page)}

Impact of alternative ad hoc, a posteriori rootings on the phylogenetic classification of archaeal biodiversity.

(A, B) Unrooted trees derived from standard evolution-models are oblivious to the root and are not fully resolved into bipartitions (i.e. some braches are polytomous rather than dichotomous), and thus preclude identification of clades and sister group relationships. With multiple, independent sets of bipartitions, the archaea are unresolved in (A), but are resolved into a distinct set of bipartitions in (B). It is common practice to add a user-specified root node (green *) a posteriori to unrooted trees, by hand, based on prior knowledge (or belief) of the investigator. Such an a posteriori rooting is necessary to determine the recency of common ancestry as well as the temporal order of key evolutionary transitions that define evolutionary groups. Five possible (of many) rootings R1-R5 are shown (see text for description). (C-J) The different possible evolutionary relationships of the archaea to other taxa, depending on the position of the root, are shown. Both the Eocyte ToL (A) and the Three-Domains ToL (F) dependent on the notion that the root should be placed at position R1 in the unrooted tree. (I) Two-Empires ToL based on the root placed at position R4. (D, E, G, H, and J) arbitrarily rooted ToL. 


\section{Figure 6 (on next page)}

Global tree of life depicting the evolutionary relationships of the major taxa of life.

(A) Phylogeny of the major taxa Archaea, Bacteria and Eukarya inferred from patterns of inheritance of functional genomic signatures. Monophyly of each major taxon and placement of Archaea sister to Bacteria supports a dichotomous classification of the diversity of life such that Archaea and Bacteria together constitute a clade of akaryotes (or Akarya). Eukarya and Akarya are sister-clades that diverge from the universal common ancestor (UCA) at the root of the tree of life. Each clade is supported by the highest posterior probability of 1.0 . The phylogeny supports a scenario of independent origins and descent of eukaryote and akaryote species. (B) Model selection tests identify, overwhelmingly, directional evolution-models to be better-fitting models to describe the evolution of genomic signatures. (C) The estimated phylogeny, especially the placement of the root is robust to both CSRH and LSRH. Alternative hypotheses, and accordingly alternative classifications or scenarios for the origins of the major clades of life, are much less probable and not supported. 


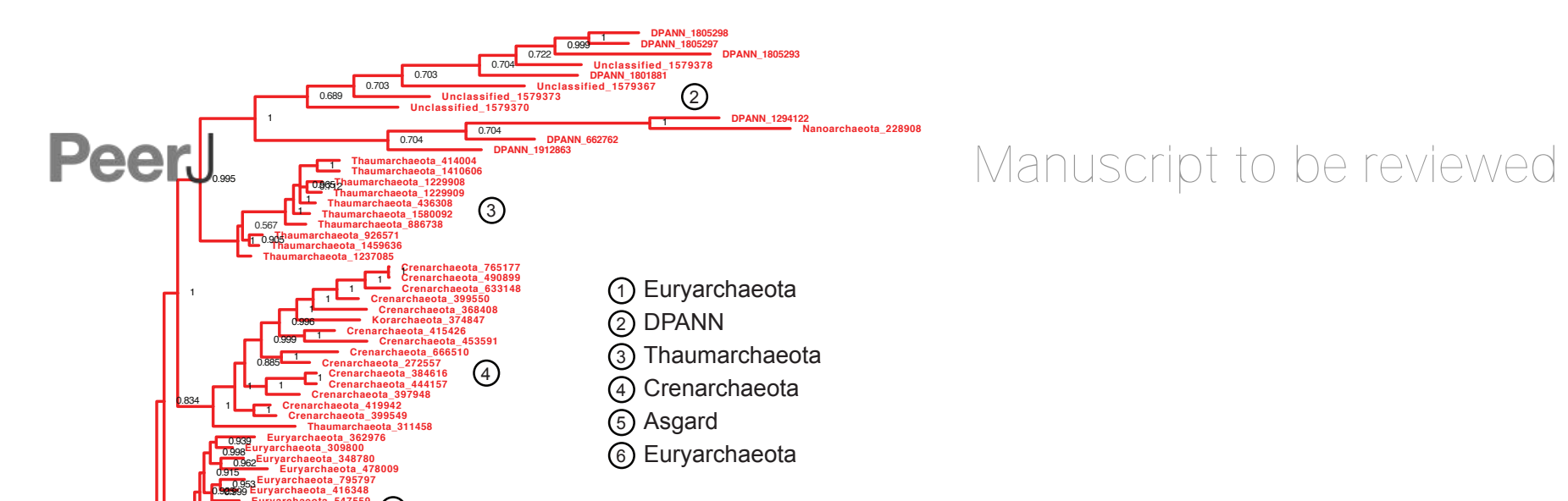

B

i. Model selection using RJ-MCMC algorithm

\begin{tabular}{|c|c|c|c|c|c|c|}
\hline Dataset & $\begin{array}{l}\text { No. of } \\
\text { characters }\end{array}$ & $\begin{array}{l}\text { Evolution } \\
\text { model }\end{array}$ & $\begin{array}{l}\text { Posterior } \\
\text { probability }\end{array}$ & $\begin{array}{l}\text { Standard } \\
\text { deviation }\end{array}$ & $\begin{array}{l}\text { Minimum } \\
\text { probability }\end{array}$ & $\begin{array}{l}\text { Maximum } \\
\text { probability }\end{array}$ \\
\hline \multirow{2}{*}{\multicolumn{2}{|c|}{ Data }} & Unpolarized & 0.501 & 0.006 & 0.497 & 0.506 \\
\hline & & Direction & 0.499 & 0.006 & 0.494 & 0.503 \\
\hline & 1,732 & Directional & 1.000 & 0.000 & 1.000 & 1.000 \\
\hline SCOP-II & 1,738 & Directional & 1.000 & 0.000 & 1.000 & 1.000 \\
\hline
\end{tabular}

ii. Model selection using a best-fit criterion

\begin{tabular}{lllll}
\hline Dataset & $\begin{array}{l}\text { No. of } \\
\text { characters }\end{array}$ & $\begin{array}{l}\text { Evolution } \\
\text { model }\end{array}$ & $\begin{array}{l}\text { Likelihood } \\
\text { score }\end{array}$ & LBF \\
\hline SCOP-II & 1,738 & $\begin{array}{l}\text { Mk+G12+Dir } \\
\text { (Directional) }\end{array}$ & -78941 & 0 \\
& $\begin{array}{l}\text { Mk+G12 } \\
\text { (Unpolarized) -79797 }\end{array}$ & 856 \\
\hline
\end{tabular}

\begin{tabular}{|c|c|c|c|c|c|c|c|c|}
\hline \multirow{8}{*}{ 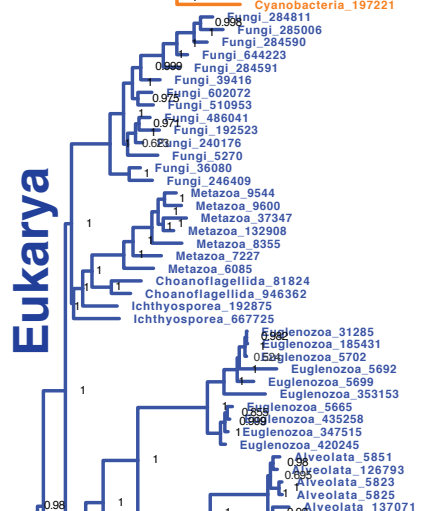 } & \multicolumn{8}{|l|}{ C } \\
\hline & Model Complexity & Evolution model & $\begin{array}{l}\text { Root } \\
\text { position }\end{array}$ & $\begin{array}{l}\text { Archaeal } \\
\text { radiation }\end{array}$ & \multirow{2}{*}{$\begin{array}{l}\text { Sister } \\
\text { group }\end{array}$} & \multirow[t]{2}{*}{$\begin{array}{l}\text { Hypothesis or } \\
\text { Scenario }\end{array}$} & \multirow[t]{2}{*}{$\begin{array}{l}\text { Likelihood } \\
\text { score }\end{array}$} & \multirow[t]{2}{*}{ LBF } \\
\hline & \multicolumn{4}{|c|}{ Relaxed-clock models } & & & & \\
\hline & \multirow{5}{*}{$\begin{array}{l}\text { LSRH } \\
\text { CSRH } \\
\text { Nonreversible } \\
\text { Nonstationary }\end{array}$} & $\overline{M k+G 12+D i r+l G R}$ & R4 & "Monophyletic & Bacteria & Two Empires & $\begin{array}{c}-78828 \\
\end{array}$ & 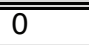 \\
\hline & & Mk+G12+Dir+CPP & $\mathrm{R} 4$ & Monophyletic & Bacteria & Two Empires & -78849 & 21 \\
\hline & & $\overline{M k+G 12+D i r+T K 02}$ & R4 & Monophyletic & Bacteria & Two Empires & -78852 & 23 \\
\hline & & Mk+G12+Dir+IGR & $\mathrm{R} 2$ & Monophyletic & $\begin{array}{c}\text { Eukarya } \\
+ \\
\text { Bacteria }\end{array}$ & Unnamed & -79035 & 206 \\
\hline & & $\overline{M k}+G 12+D i r+I G R$ & R1 & Monophyletic & Eukarya & Three Domains & -79038 & 210 \\
\hline 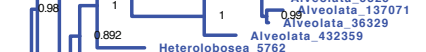 & \multicolumn{8}{|c|}{ Non-clock models } \\
\hline 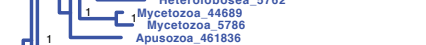 & \multirow{5}{*}{$\begin{array}{l}\text { CSRH } \\
\text { Nonreversible } \\
\text { Nonstationary }\end{array}$} & $\overline{\mathrm{Mk}+\mathrm{G} 12+\mathrm{Dir}}$ & $\overline{\mathrm{R} 4}$ & Monophyletic & Bacteria & Two Empires & $\begin{array}{l}-78941 \\
\end{array}$ & 0 \\
\hline 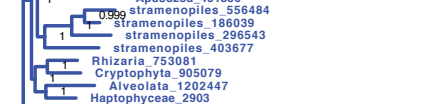 & & Mk+G12+Dir & R2 & Monophyletic & $\begin{array}{c}\text { Eukarya } \\
+ \\
\text { Bacteria }\end{array}$ & Unnamed & -79276 & 336 \\
\hline 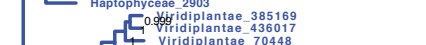 & & $\overline{M k+G 12+D i r}$ & R1 & Monophyletic & Eukarya & Three Domains & -79334 & 394 \\
\hline 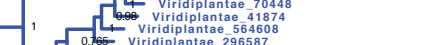 & & $\mathrm{Mk}+\mathrm{G} 12+\mathrm{Dir}$ & R3 & Paraphyletic & NA & Unnamed & -79508 & 567 \\
\hline 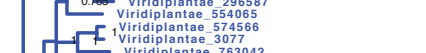 & & Mk+G12+Dir & R1 & Paraphyletic & Bacteria & Eocyte & -79696 & 755 \\
\hline 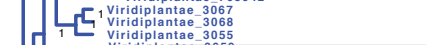 & \multirow{2}{*}{\multicolumn{2}{|c|}{ 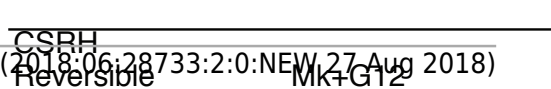 }} & & & & & & \\
\hline 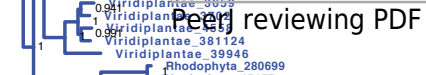 & & & Unrooted & - & - & - & -79797 & 856 \\
\hline 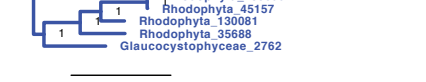 & $\begin{array}{l}\text { Reversible } \\
\text { Stationary } \\
\end{array}$ & Mk & Unrooted & - & - & - & -85817 & 6876 \\
\hline
\end{tabular}




\section{Table $\mathbf{1}$ (on next page)}

Phylogenomic datasets that use different character types, and the source of the datasets; SCOP $=$ Structural Classification of Proteins. 


\section{Table 1}

2

\begin{tabular}{lllll} 
Dataset & Character type & no. of taxa & no. of loci & no. of characters \\
SSU rRNA $^{\mathrm{a}}$ & Elementary (nucleotide) & 140 & 1 gene & 1,462 \\
Core-genes-I $^{\mathrm{b}}$ & Elementary (amino acid) & 44 & 29 genes & 8,563 \\
Core-genes-II $^{\mathrm{a}}$ & Elementary (amino acid) & 96 & 48 genes & 9,868 \\
SCOP-domains-I $^{\mathrm{c}}$ & Complex (protein domain) & 141 & 1,732 domains & 1,732 \\
SCOP-domains-II $^{\mathrm{d}}$ & Complex (protein domain) & 222 & 1,738 domains & 1,738 \\
\hline
\end{tabular}

a(Zaremba-Niedzwiedzka, K., Caceres, E.F., et al. 2017) ; b(Williams, T.A. and Embley, T.M. 2014) ; c(Harish, A., Tunlid, A., et al. 2013) ; ${ }^{\mathrm{d}}$ present study 


\section{Table 2 (on next page)}

Redundant representation of genomic loci (protein-domains) in concatenated coregenes datasets.

The P-loop NTP hydrolase domain is one of the most prevalent domains. Genomic loci encoding P-loop hydrolase domain are represented 8-9 times in each species in the singlecopy genes employed in the core-genes datasets. Redundant loci in the core-genes datasets vary depending on the genes and species sampled for phylogenomic analyses. In contrast, SCOP-domain datasets are composed of unique loci. 
Table 2.

\begin{tabular}{|c|c|c|c|c|c|c|c|}
\hline Dataset & $\begin{array}{l}\text { No. } \\
\text { of } \\
\text { tax } \\
\text { a } \\
\end{array}$ & $\begin{array}{l}\text { No. of } \\
\text { uniqu } \\
\text { e } \\
\text { genes }\end{array}$ & $\begin{array}{l}\text { No. of } \\
\text { unique } \\
\text { domain } \\
\text { s }\end{array}$ & & Redundant domains & $\begin{array}{l}\text { No. of } \\
\text { times } \\
\text { redunda } \\
\text { nt in } \\
\text { each } \\
\text { taxon }\end{array}$ & $\begin{array}{l}\text { No. of } \\
\text { taxa in } \\
\text { which } \\
\text { redunda } \\
\text { nt }\end{array}$ \\
\hline \multirow{17}{*}{$\begin{array}{l}\text { Core- } \\
\text { genes-I }\end{array}$} & \multirow{17}{*}{44} & \multirow{17}{*}{29} & \multirow{17}{*}{35} & $\begin{array}{l}\text { SCOP } \\
\text { Uniqu } \\
\text { e ID }\end{array}$ & Description & & \\
\hline & & & & \multirow{5}{*}{52540} & \multirow{5}{*}{ P-loop containing NTP hydrolases } & 9 & 33 \\
\hline & & & & & & 8 & 4 \\
\hline & & & & & & 7 & 3 \\
\hline & & & & & & 6 & 2 \\
\hline & & & & & & 5 & 2 \\
\hline & & & & \multirow{3}{*}{50447} & \multirow{3}{*}{ Translation proteins } & 4 & 8 \\
\hline & & & & & & 3 & 26 \\
\hline & & & & & & 2 & 9 \\
\hline & & & & \multirow{2}{*}{54211} & \multirow{2}{*}{ Ribosomal protein S5 domain 2-like } & 3 & 42 \\
\hline & & & & & & 2 & 2 \\
\hline & & & & \multirow{2}{*}{50249} & \multirow{2}{*}{ Nucleic acid-binding proteins } & 3 & 38 \\
\hline & & & & & & 2 & 5 \\
\hline & & & & 53067 & Actin-like ATPase domain & 2 & 16 \\
\hline & & & & 54980 & EF-G C-terminal domain-like & 2 & 42 \\
\hline & & & & 64484 & $\begin{array}{l}\text { beta and beta-prime subunits of DNA dependent RNA- } \\
\text { polymerase }\end{array}$ & 2 & 41 \\
\hline & & & & 47364 & Domain of the SRP/SRP receptor G-proteins & 2 & 2 \\
\hline \multirow{3}{*}{$\begin{array}{c}\text { Core- } \\
\text { genes-II }\end{array}$} & \multirow{3}{*}{96} & \multirow{3}{*}{48} & \multirow{3}{*}{50} & \multirow{3}{*}{50249} & \multirow{3}{*}{ Nucleic acid-binding proteins } & 5 & 3 \\
\hline & & & & & & 4 & 81 \\
\hline & & & & & & 3 & 11 \\
\hline
\end{tabular}




\begin{tabular}{|c|c|c|c|c|c|c|c|}
\hline & & & & & & 2 & 1 \\
\hline & & & & 50104 & Translation proteins $\mathrm{SH} 3$-like domain & 3 & 78 \\
\hline & & & & 50104 & Iranstation protems sHs-nke domam & 2 & 18 \\
\hline & & & & 50447 & Tronclation protainc & 3 & 15 \\
\hline & & & & 30441 & 1ranstationt piotemins & 2 & 71 \\
\hline & & & & 64484 & beta and beta-prime subunits of DNA dependent RNA- & 3 & 88 \\
\hline & & & & 04484 & polymerase & 2 & 5 \\
\hline & & & & 52540 & P-loop containing NTP hydrolases & 2 & 83 \\
\hline & & & & 53067 & Actin-like ATPase domain & 2 & 40 \\
\hline & & & & 53137 & Translational machinery components & 2 & 90 \\
\hline & & & & 54211 & Ribosomal protein S5 domain 2-like & 2 & 93 \\
\hline & & & & 56053 & Ribosomal protein L6 & 2 & 88 \\
\hline SCOP-I & 141 & - & 1,732 & - & - & - & - \\
\hline $\begin{array}{l}\text { SCOP- } \\
\text { II }\end{array}$ & 222 & - & 1,738 & - & - & - & - \\
\hline
\end{tabular}

3 